\title{
WILLIAM ROXBURGH'S PLANTS OF THE COAST OF COROMANDEL: AN ENUMERATION OF SPECIES
}

\author{
K.M. MATTHEW $\dagger$ \\ Late of the Rapinat Herbarium, St. Joseph's College, Tiruchirapalli, India, obit. 16th March 2004. \\ Prepared for publication and edited by \\ H.J. Noltie, Royal Botanic Garden, EH3 5LR Edinburgh, United Kingdom
}

\section{SUMMARY}

The scope of the present work is to provide, for the first time, a road map to the contents of Roxburgh's great illustrated work by means of a systematic enumeration of the species, with a note on the provenance of the material. An introduction is given of Roxburgh's life and work.

\section{INTRODUCTION}

\section{LIFE AND WORK}

William Roxburgh's life has been extensively documented by King (1895), Burkill (1965) and Desmond (1992), and most recently by Robinson (2003), but it seems worthwhile giving a summary here. His early life is poorly documented, but he was born in Ayrshire in 1751 and studied medicine at the University of Edinburgh in 1771/1772, where he attended John Hope's botanical lectures. In 1776 he became an Assistant Surgeon on the Madras Establishment of the East India Company (EIC). He made the acquaintance of J.G. König of the Danish establishment at Tranquebar (who had arrived in 1768) and eventually became Government botanist to the EIC. König's botanical work impressed the Madras Government enough to allow him to make collections as far afield as Siam and the Straits of Malacca. However, his health deteriorated rapidly and he died at Tranquebar in 1785 ministered to by Roxburgh himself.

Roxburgh moved to Samulcotah, "a small station about seven miles from the town of Coconada, and about twenty-two miles from one of the mouths of the Godavery river" (King, 1895: 2), in 1781. Here he established a garden for the growing and investigation of economic plants, and explored the surrounding area of the Northern Circars. In 1793 he moved to Calcutta where he was appointed to succeed Colonel Robert Kyd in charge of the EIC's Botanic Garden; he remained here until 1813, with two long sick leaves at the Cape of Good Hope (1798-1799) and in Britain (1805-1807). He finally left Calcutta in 1813, his health by this time shattered, and, via the Cape of Good Hope and St. Helena, reached Edinburgh where he died in 1815.

Roxburgh was renowned for his field work, the accuracy of his descriptions and especially for the quality of work he obtained from native artists in the execution of several thousand plant illustrations. Unfortunately, the extensive plant collections he made were not generally available to later workers, rendering the typification of his 


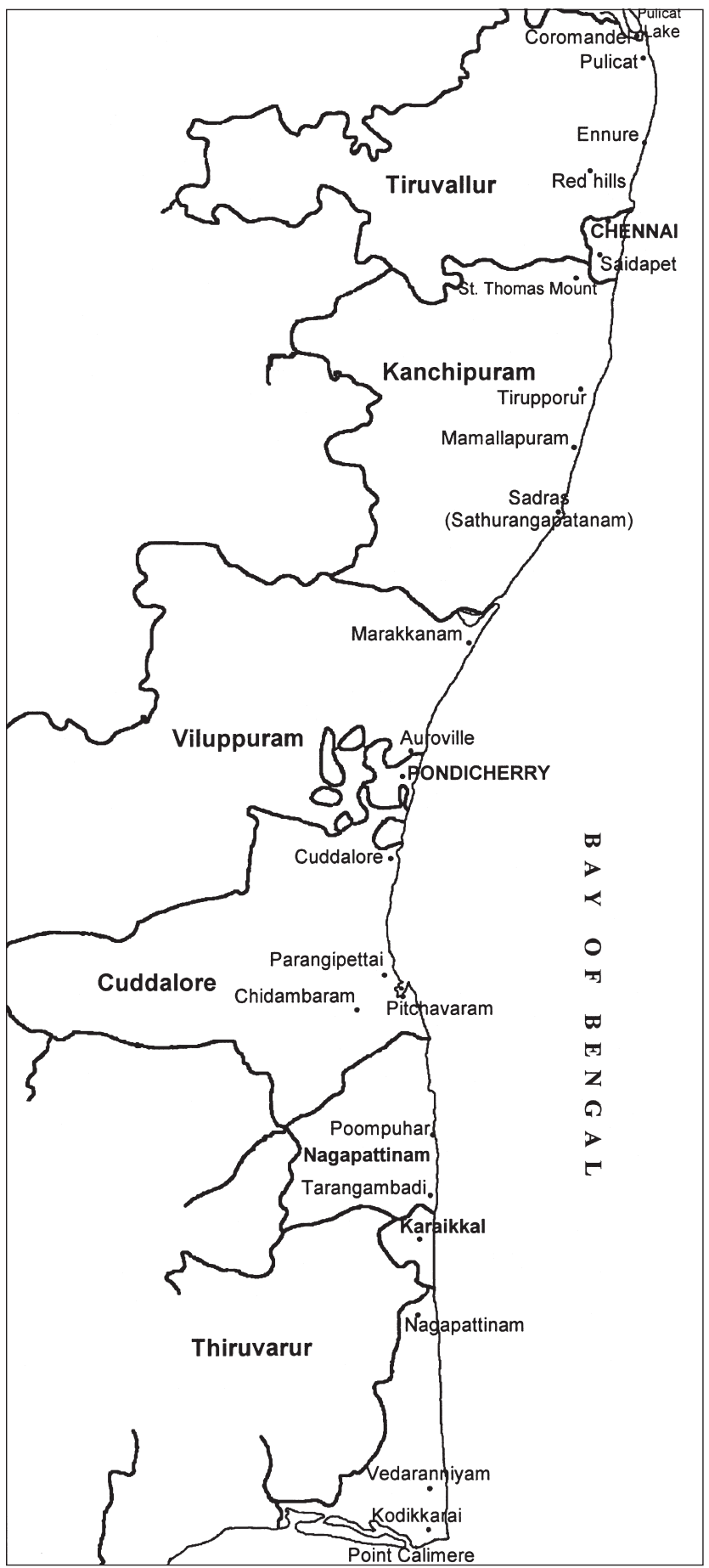

Map 1. Coromandel coast. 
plant names problematic (see below). His early collections from the northern Circars were lost in a flood at Coringa in 1787; those made from Calcutta were, according to William Griffith, distributed to various European herbaria by Nathaniel Wallich, but Wallich himself strenuously denied this. Whatever the truth of this the extant collections are widely scattered, at Geneva, Brussels, Kew, London (Natural History Museum \& Linnean Society), Liverpool, Edinburgh and Oxford. For further details see Forman (1997).

Because of the sparse details on the extant specimens and their wide dispersal, the paintings he commissioned (known as the Roxburgh Icones) are indispensable for the typification of Roxburgh's plant names. Several sets exist, the one at Kew having been documented by Sealy $(1956,1975)$ and that at Calcutta by Sanjappa et al. (1993). Considerable biographical information emanating from Roxburgh's vast correspondence has come from Edinburgh (Hedge \& Lamond, 1989; Robinson, 2003) and Calcutta (Nayar \& Das, 1984, 1985).

The Plants of the Coast of Coromandel (1795-1820), his most spectacular publication, was also the first published book devoted to the plants of the east coast of India. Its origins are intimately connected with the EIC's policy of economic exploitation of the plant resources of the country. Patrick Russell, a Company physician who had arrived in 1782, was impressed by the work of König and Roxburgh, and wrote to Sir Joseph Banks, President of the Royal Society in London, suggesting the idea of preparing illustrations of the more important plants. With König's death in 1785, the job was entrusted to Russell who, however, left the country in 1789 . Thus the job finally fell to Roxburgh who had by now acquired considerable knowledge of native plants with his work in Northern Circars (1781-1793). Sir Joseph Banks received the first consignment of plates from Roxburgh in 1790. Printing was started in 1794. The first fascicle of 25 plates appeared in 1795 and the series of 300 plates was completed by 1820 in 12 fascicles (Desmond, 1977, 1992; Stafleu \& Cowan, 1983: 954-958).

\section{RELATIVE OBLIVION}

The book was a landmark publication (Anon., 1823) for its intrinsic worth: high quality illustrations, description of many novelties, extensive information on the uses of plants, manner of extraction of plant products and copious local information including vernacular names. However, it passed into oblivion for two reasons.

Firstly, it was formidably expensive, and the size of the plates ( 58 by $46 \mathrm{~cm}$ ) made it unwieldy. The plates formed an impressive florilegium that, indeed, succeeded in persuading the Directors of the East India Company in favour of their publication, but they were certainly not handy for general use. Having advanced $£ 2,000$ up to 1799 , the Company's receipts were only $£ 401$. Distribution of a large number of complimentary copies might have been one reason for this; and none of the 40 plain copies sent to India (at 20 Rupees per copy) was sold. Finally, the refusal by the Royal Botanic Gardens Kew of the offer of 194 of the original copper etching plates by the India Office in 1881 sealed the fate of the book (Desmond, 2003). The volumes remained locked up in big libraries, without even a comprehensive analytical index. The book has been reissued both in microfiche and 'hard' copy (on a reduced scale by Bishen Singh Mahendra Pal Singh, 1981), but even so it has been to a large extent overlooked. 
Secondly, it dealt with only a tiny proportion of the Indian flora, some 300 plants, and publication took a quarter of a century to complete (Wood, 1969). Its value was reduced with the publication of its botanical content in the author's unillustrated Flora Indica (Roxburgh, 1820, 1824, 1832) and was, to a large extent, superseded by the great Flora of British India (Hooker, 1872-1897). The production of over two thousand plates of Indian plants at affordable prices and in slightly more portable format (Wight, 1838-1853; Beddome, 1874) also somewhat overshadowed Roxburgh's pioneering, if extravagant, effort.

\section{'COROMANDEL': TWO LITTLE KNOWN ANOMALIES}

1. Neither the derivation of the name, nor even the location of the place, is certain. As to the name 'Coromandel Coast', the Imperial Gazetteer of India (Thornton, 1886: 205) reports the following: "Part of the eastern coast of Southern India, forming the shore of the Bay of Bengal. It is considered to commence at Point Calimere $\left(10^{\circ} 17^{\prime}\right.$ $\left.\mathrm{N}, 79^{\circ} 56^{\prime} \mathrm{E}\right)$, and to hold a direction nearly due north as far as Gondegam $\left(15^{\circ} 20^{\prime} \mathrm{N}\right.$, $\left.80^{\circ} 10^{\prime} \mathrm{E}\right)$... The etymology of the name Coromandel has been variously explained, some tracing it to Karimanal ... but it appears that it was originally denominated Choramandal or Cholamandal, which is considered to mean the mandal or region of the Chola, an ancient dynasty of this part of India". And, as to the location of a settlement of this name (Map 1): "Town in Chengalpat district, Madras, $13^{\circ} 26^{\prime} 10^{\prime \prime} \mathrm{N}$, $80^{\circ} 20^{\prime} 36^{\prime \prime}$ E ... Karimanal or black sand commonly used as pounce, is found here". The name 'Coromandel' has recently disappeared from the map since the people of the entire village have recently been relocated to make room for the Space Research Station of the Government of India at Sri Harikota.

2. The use of the term 'Coromandel Coast' for the title of the work under consideration is not entirely appropriate. The name refers to the region where Roxburgh began his professional career in 1776 (Tranquebar-Madras sector, see Map 1). The early botanical pioneers from J.G. König onwards were based at Tranquebar, the headquarters of the Danish Christian Mission. And commercial activity was centred around Madras, some $250 \mathrm{~km}$ to its north. But Roxburgh shifted from this region to Samulcotah in 1781 over $300 \mathrm{~km}$ to the north of Madras, far beyond the northern boundary of the "Coromandel Coast', when supervising the work of the EIC's Gardens there. In 1793 he shifted to Calcutta, another $600 \mathrm{~km}$ to the north, where he was in charge of the Company's much larger and more important Garden (1793-1813). The plants dealt with in the book did not belong to as limited a geographical area as the title would suggest, but were primarily plants of economic importance, originating from the following broad regions (see Table 1 for details): i) East Coast of India (Roxburgh's 'Coromandel' coast), mostly from the present State of Andhra Pradesh ('Circars' region) (197); ii) North and East India (56); iii) South India (19); iv) India (general) (14); v) Other countries (21).

\section{THE PRESENT SCOPE}

The taxonomic significance of the Plants of the Coast of Coromandel lies in the assistance it can give in the typification of Roxburgh's plant names, especially in the absence 
Table 1. Provenance of plants.

Note. The purpose of this is to draw attention to the geographically wide range of plant sources of the plants described. Because of the sketchy nature of the details given by Roxburgh, this is to be regarded as no more than an approximation: many have no details (though their origin may often be guessed), and some species are described as coming from more than one place.

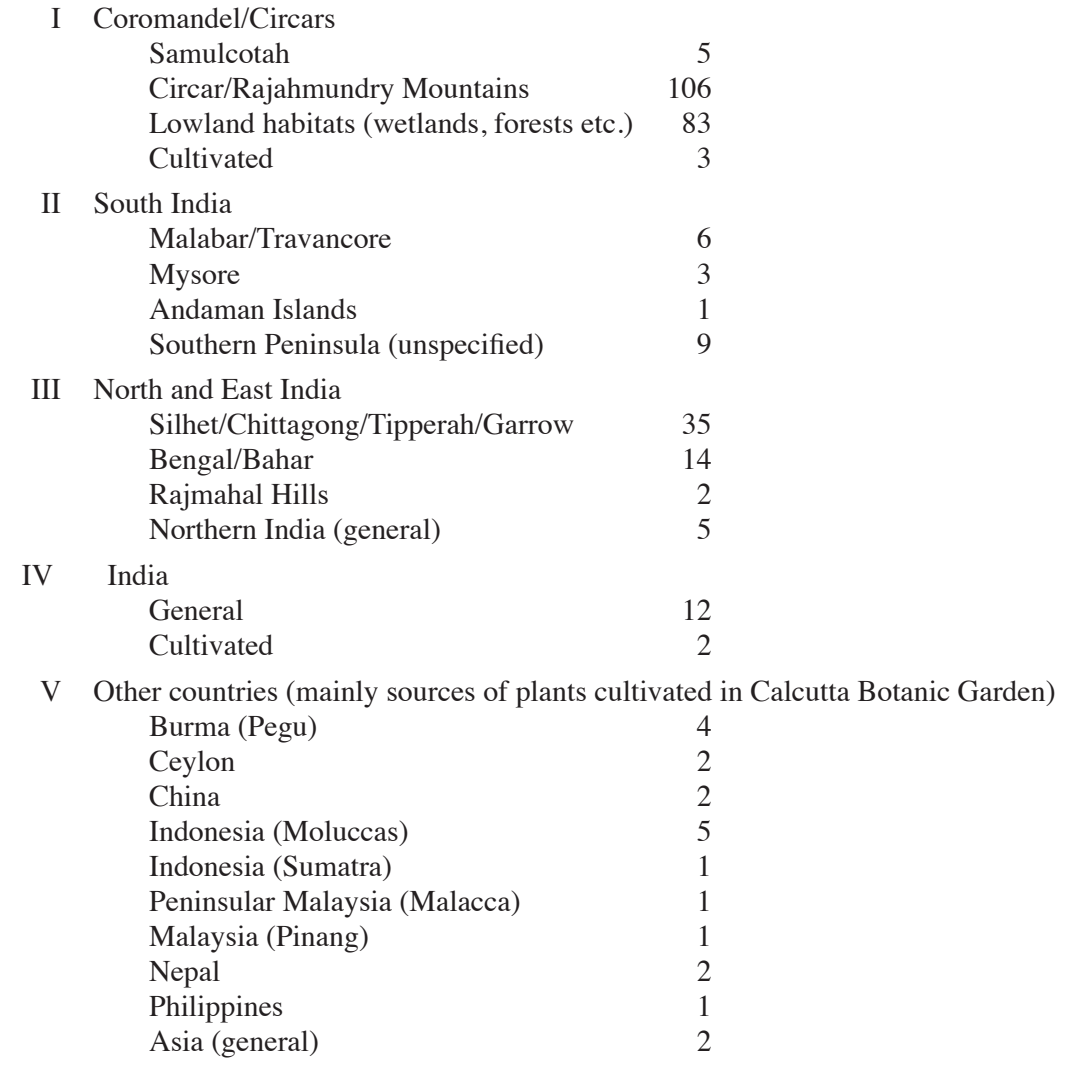

of specimens, or, when extant, which lack data. The pioneering efforts of Merrill (1952, unpublished) have been carried forward significantly by Forman (1997) whose account is so thorough, both in indicating the known locations of Roxburgh specimens (and drawings), and in pointing out the problems of typification, that it will remain the starting point for any further work on the subject. However, even in Forman's list only some 80 out of the 300 Coromandel plants are referred to. Two other sources of information are Morton (1974) and Bretschneider (1898: 237-246), the latter with regard to the Chinese materials.

The scope of the present work is to provide, for the first time, a road map to the contents of the work by means of a systematic enumeration of the species, with a note on the provenance of the material. 
i) Nomenclature: The name of the plant as it appeared in 'Coromandel Plants' appears first (sometimes an earlier name was adopted by Roxburgh, but not always correctly), without further references, even to Roxburgh's own later works. This procedure keeps the focus sharply on Roxburgh himself. If this name is now considered the correct one, bold face is used; otherwise, italics. The reference to 'Coromandel Plants' has four elements: volume, column (NB: there are two columns per page), plate number and year, respectively. This 'Coromandel Plants'-based citation should sharpen attention on the need of typification of Roxburgh's names which is the ultimate aim of this paper, as already done for the work of another early south Indian botanist, J.P. Rottler (Matthew, 1993). Those marked 'nom. illeg.' are superfluous.

The name now considered correct, if different from the above, appears in bold face in a new paragraph. Basionyms are cited when necessary to explain any double citation.

ii) Provenance (see Table 1): These brief notes are important in giving the source of the plants described, and in drawing attention to their heterogeneous scope. They came from two main areas, based around Roxburgh's superintendentship of the EIC's gardens, first at Samulcotah (1781-1793) and later at Calcutta (1793-1813). Eventually the entire work revolved around Calcutta, where economic plants were cultivated under Roxburgh's supervision in his later years.

Plants were brought to Calcutta from various parts of India and even abroad. Within India, the Province of Bengal and the species-rich north-eastern regions of the Indian subcontinent (including Silhet and Chittagong now in Bangladesh) were the main sources; others were the Gangetic plains, Mysore, Kerala and the Andaman Islands.

Table 2. Gazetteer of localities

Roxburgh's spelling of place names has been retained in the Enumeration. The following gives their current equivalents.

\begin{tabular}{ll}
\hline Roxburgh & Current \\
\hline Bahar & Bihar State \\
Calcutta & Kolkota (West Bengal) \\
Ceylon & Sri Lanka \\
Circars & Now part of Andhra Pradesh State \\
Coconada & Kakinada (Andhra Pradesh) \\
Garrow Hills & Garo Hills (Meghalaya) \\
Golparah & Goalpara (Assam) \\
Madras & Chennai (Tamil Nadu) \\
Malabar & SW coast, now in Kerala State \\
Moluccas & Maluku (Indonesia) \\
Mysore & Former princely state, now part of Karnataka State \\
Pulo-Pinang & Pinang Island (Malaysia) \\
Samulcotah & Samalkot (Andhra Pradesh) \\
Tanjour & Thanjavur (Tamil Nadu) \\
Tipperah & Tripura (State in NE India) \\
Tranquebar & Tarangambadi (Tamil Nadu) \\
\hline
\end{tabular}


Other countries involved were Nepal, Ceylon, Burma, Malaysia, Indonesia and the Philippines. Plants from the Calcutta Botanic Garden are well localized, with information on the source of introduction of the plant, etc. (Roxburgh, 1814).

A second group of plants came from around Samulcotah, where Roxburgh's acquaintance with the vegetation of the country originated. This group has a significance of its own, for its stamp of firsthand knowledge of local plants and the country, as is apparent from the high quality field observations on species from the Circars region.

Finally, there is a third group of species with no obvious economic importance, such as Scrophulariaceae. These carry little field information and were no doubt mainly described and illustrated from the Circars.

\section{SOME CONVENTIONS}

1. Place names are spelt as by Roxburgh, modern equivalents are given in Table 2 .

2. Provenance (Table 1). For the non-localised specimens, some idea of their origin can be obtained from the native names given by Roxburgh. Those with a 'Telinga' (i.e. Telugu) name no doubt come from the Circars of Andhra Pradesh and those with a 'Tamul' (i.e. Tamil) name from the area around Madras in Tamil Nadu. These regions have been added in square brackets.

\section{ENUMERATION OF SPECIES}

\section{ACANTHACEAE}

Justicia acaulis L.f., Suppl. Pl. (1782) 84; Roxb., Pl. Coromandel 2 (1800) 15, t. 127. Elytraria acaulis (L.f.) Lindau in Engl. \& Prantl, Nat. Pflanzenfam. 1, 163 (1897) 304.

Provenance: Pasture ground, generally under the shade of trees [Circars].

Justicia montana Roxb., Pl. Coromandel 2 (1805) 41, t. 176.

Eranthemum capense L., Sp. Pl. (1753) 9.

Provenance: Mountains.

Justicia pulchella (Andrews) Roxb., Pl. Coromandel 2 (1805) 41, t. 177.

Eranthemum pulchellum Andrews, Bot. Repos. (1800) t. 88.

This is a legitimate new combination, but Roxburgh misinterpreted Andrews's name and the correct name for Roxburgh's plant is:

Eranthemum purpurascens Nees in Wall., Pl. Asiat. Rar. 3 (1832) 106. Provenance: Dry, uncultivated, shady places.

Lepidagathis cristata Willd., Sp. Pl. 3 (1800) 400; Roxb., Pl. Coromandel 3 (1820) 63, t. 267.

Provenance: Barren ground, Coast of Coromandel.

Justicia pectinata L., Amoen. Acad. 4 (1759) 209; Roxb., Pl. Coromandel 2 (1802) 29, t. 153.

Rungia pectinata (L.) Nees in DC., Prodr. 11 (1847) 469.

Provenance: Shady places. 
Justicia repens L., Sp. Pl. (1753) 15; Roxb., Pl. Coromandel 2 (1802) 29, t. 152.

Rungia repens (L.) Nees in Wall., Pl. Asiat. Rar. 3 (1832) 110.

Provenance: Pasture ground.

Thunbergia fragrans Roxb., Pl. Coromandel 1 (1796) 47, t. 67.

Provenance: Banks of watercourses, about Samulcotah.

\section{AIZOACEAE}

Gisekia pharnaceoides L., Mant. Pl. (1771) 562; Roxb., Pl. Coromandel 2 (1805) 43, t. 183.

Provenance: Cultivated ground [Circars].

\section{ALANGIACEAE}

Marlea begonifolia Roxb., Pl. Coromandel 3 (1820) 80, t. 283.

Alangium chinense (Lour.) Harms, Ber. Deutsch. Bot. Ges. 15 (1897) 24.

Stylidium chinense Lour., Fl. Cochinch. 1 (1790) 221.

Provenance: Silhet.

\section{ANACARDIACEAE}

Buchanania angustifolia Roxb., Pl. Coromandel 3 (1820) 58, t. 262.

Buchanania axillaris (Desr.) Ramamoorthy in Saldanha \& Nicolson, Fl. Hassan Dist. (1976) 374.

Mangifera axillaris Desr. in Lam., Encycl. 3 (1792) 697.

Provenance: Cultivated in Calcutta Botanic Garden from seed from southern part of the peninsula of India.

Holigarna longifolia Roxb., Pl. Coromandel 3 (1820) 79, t. 282.

Provenance: Chittagong hills and Malabar.

Semecarpus anacardium L.f., Suppl. Pl. (1782) 182; Roxb., Pl. Coromandel 1 (1795) 13, t. 12.

Provenance: Mountainous parts of India.

\section{ANNONACEAE}

Uvaria lutea Roxb., Pl. Coromandel 1 (1795) 32, t. 36.

Alphonsea lutea (Roxb.) Hook.f. \& Thomson, Fl. Ind. (1855) 153.

Provenance: Mountains [Circars].

Unona longiflora Roxb., Pl. Coromandel 3 (1820) 87, t. 290.

Desmos longiflorus (Roxb.) Saff., Bull. Torrey Bot. Club 39 (1912) 507.

Provenance: Silhet.

Uvaria tomentosa Roxb., Pl. Coromandel 1 (1795) 31, t. 35.

Miliusa tomentosa (Roxb.) Finet \& Gagnep., Bull. Soc. Bot. France 53 (1906) 153.

Provenance: Circar mountains. 
Uvaria cerasoides Roxb., Pl. Coromandel 1 (1795) 30, t. 33.

Polyalthia cerasoides (Roxb.) Bedd., Fl. Sylv. S. India (1869) t. 1.

Provenance: Circar mountains.

Uvaria suberosa Roxb., Pl. Coromandel 1 (1795) 31, t. 34.

Polyalthia suberosa (Roxb.) Thwaites, Enum. Pl. Zeyl. (1864) 398.

Provenance: Mountains [Circars].

\section{ANTHERICACEAE}

Anthericum tuberosum Roxb., Pl. Coromandel 2 (1800) 20, t. 138.

Chlorophytum tuberosum (Roxb.) Baker, J. Linn. Soc., Bot. 15 (1876) 332.

Provenance: Moist valleys among the Circar mountains.

\section{APOCYNACEAE}

Echites grandiflora Roxb., Pl. Coromandel 3 (1820) 78, t. 281.

Beaumontia grandiflora (Roxb.) Wall., Tent. Fl. Napal. (1824) t. 7.

Provenance: Hilly parts of Chittagong and Silhet.

Carissa carandas L., Mant. Pl. (1767) 52; Roxb., Pl. Coromandel 1 (1798) 55, t. 77. Provenance: Most wild, woody, dry, uncultivated parts [of India].

Willughbeia edulis Roxb., Pl. Coromandel 3 (1820) 77, t. 280.

Provenance: Chittagong, Silhet.

\section{APONOGETONACAE}

Aponogeton monostachyon L.f., Suppl. Pl. (1782) 214; Roxb., Pl. Coromandel 1 (1798) 58, t. 81 .

Aponogeton natans (L.) Engl. \& K. Krause in Engl., Pflanzenr. IV.13 (Heft 24) (1906) 11.

Saururus natans L., Mant. Pl. (1771) 227.

Provenance: Shallow, standing, sweet water [Circars].

\section{ARACEAE}

Arum campanulatum Roxb., Pl. Coromandel 3 (1820) 68, t. 272, nom. illeg. (includes type of A. paeoniifolium Dennst.).

Amorphophallus paeoniifolius (Dennst.) Nicolson, Taxon 26 (1977) 338.

Arum paeoniifolium Dennst., Schlüssel Hortus Malab. 13 (1818) 38.

Provenance: Woods near Calcutta (wild); Northern Circars (cultivated).

Ambrosinia ciliata Roxb., Pl. Coromandel 3 (1820) 90, t. 294.

Cryptocoryne ciliata (Roxb.) Fisch. ex Wydler, Linnaea 5 (1830) 428.

Provenance: Wet banks of rivulets, ponds, etc. near Calcutta. 
Pistia stratiotes L., Sp. Pl. (1753) 963; Roxb., Pl. Coromandel 3 (1820) 64, t. 268.

Provenance: Pools of stagnant water over most parts of India.

\section{ASCLEPIADACEAE}

Stapelia adscendens Roxb., Pl. Coromandel 1 (1795) 28, t. 30.

Caralluma adscendens (Roxb.) Haw., Syn. Pl. Succ. (1812) 47.

Provenance: On high, dry, barren ground [Circars].

Stapelia umbellata (Haw.) Roxb., Pl. Coromandel 3 (1815) 36, t. 241.

Caralluma umbellata Haw., Syn. Pl. Succ. (1812) 47.

Provenance: Cultivated in Calcutta Botanic Garden from Mysore.

Ceropegia bulbosa Roxb., Pl. Coromandel 1 (1795) 11, t. 7.

Provenance: Dry, barren, uncultivated ground [Circars].

Ceropegia acuminata Roxb., Pl. Coromandel 1 (1795) 12, t. 8.

Ceropegia bulbosa Roxb. var. lushii (Graham) Hook.f., Fl. Brit. India 4 (1883) 68.

Ceropegia lushii Graham in Curtis's Bot. Mag. (1834) t. 3300.

Provenance: Dry, barren, uncultivated ground [Circars].

Ceropegia tuberosa Roxb., Pl. Coromandel 1 (1795) 12, t. 9.

Ceropegia candelabrum L., Sp. Pl. (1753) 211.

Provenance: Dry, uncultivated ground [Circars].

Ceropegia juncea Roxb., Pl. Coromandel 1 (1795) 12, t. 10.

Provenance: Dry, uncultivated ground [Circars].

Asclepias tingens Roxb., Pl. Coromandel 3 (1815) 34, t. 239.

Gymnema tingens (Roxb.) Spreng., Syst. Veg. 1 (1824) 844.

Provenance: Cultivated in Calcutta Botanic Garden from Pegu (Burma).

Asclepias tenacissima Roxb., Pl. Coromandel 3 (1815) 35, t. 240.

Marsdenia tenacissima (Roxb.) Moon, Cat. Pl. Ceylon (1824) 21.

Provenance: Cultivated in Calcutta Botanic Garden [from the Rajmahal Hills, Bihar

- see Roxburgh, 1814: 21].

Periploca esculenta L.f., Suppl. Pl. (1782) 168; Roxb., Pl. Coromandel 1 (1795) 13, t. 11.

Oxystelma esculentum (L.f.) R.Br. ex Schult., Syst. Veg. 6 (1820) 89.

Provenance: Banks of watercourses, pools, etc. [Circars].

\section{BIGNONIACEAE}

Bignonia spathacea auct. non L.f. (1781); Roxb., Pl. Coromandel 2 (1800) 24, t. 144. Dolichandrone falcata (Wall. ex DC.) Seem., Fl. Vill.-Nov. (1880) 151.

Spathodea falcata Wall. ex DC., Prodr. 9 (1845) 206.

Provenance: Forests over the [Coromandel] Coast. 
Bignonia quadrilocularis Roxb., Pl. Coromandel 2 (1800) 24, t. 145.

Heterophragma quadrilocularis (Roxb.) K. Schum. in Engl. \& Prantl, Nat. Pflanzenfam. IV.3B (1895) 243.

Provenance: Circar mountains.

Bignonia suberosa Roxb., Pl. Coromandel 3 (1811) 11, t. 214, nom. illeg.

Millingtonia hortensis L.f., Suppl. Pl. (1782) 291.

Provenance: Cultivated in Calcutta Botanic Garden from the Rajah of Tanjour's garden (via Madras).

\section{BOMBACACEAE}

Bombax heptaphyllum auct. non L. (1763); Roxb., Pl. Coromandel 3 (1815) 43, t. 247.

Bombax ceiba L., Sp. Pl. (1753) 511, in part, quoad plantas asiaticas excl. syn.; Robyns, Taxon 10 (1961) 157 \& Bull. Jard. Bot. État 33 (1963) 88.

Provenance: 'Almost everywhere' [i.e. in India].

\section{BORAGINACEAE}

Ehretia buxifolia Roxb., Pl. Coromandel 1 (1796) 42, t. 57, nom. illeg.

Carmona retusa (Vahl) Masam., Trans. Nat. Hist. Soc. Taiwan 30 (1940) 61.

Cordia retusa Vahl, Symb. Bot. 2 (1791) 42.

Provenance: Barren lands and forests [Circars].

Cordia monoica Roxb., Pl. Coromandel 1 (1796) 43, t. 58.

Provenance: Forests where barren land predominates [Circars].

Ehretia aspera Willd., Phytographia 4 (1794) t. 2; Roxb., Pl. Coromandel 1 (1796) 41, t. 55.

Provenance: Dry, rocky, barren places [Circars].

Ehretia laevis Roxb., Pl. Coromandel 1 (1796) 42, t. 56.

Provenance: Circar mountains.

\section{BURMANNIACEAE}

Burmannia disticha L., Sp. Pl. (1753) 287; Roxb., Pl. Coromandel 3 (1815) 37, t. 242.

Provenance: Ceylon.

\section{BURSERACEAE}

Boswellia glabra Roxb., Pl. Coromandel 3 (1811) 4, t. 207.

Boswellia serrata Roxb. ex Colebr., Asiat. Res. 9 (1807) 379, t. 5.

Provenance: Highest mountains on the Coast of Coromandel.

Garuga pinnata Roxb., Pl. Coromandel 3 (1811) 5, t. 208.

Provenance: Various mountainous districts over India. 


\section{CAPPARACEAE}

Roydsia suaveolens Roxb., Pl. Coromandel 3 (1820) 87, t. 289.

Stixis suaveolens (Roxb.) Baill., Bull. Soc. Linn. Paris 1 (1887) 655.

Provenance: Silhet.

\section{CELASTRACEAE}

Hippocratea arborea Roxb., Pl. Coromandel 3 (1811) 3, t. 205.

Reissantia arborea (Roxb.) H. Hara, J. Jap. Bot. 40 (1965) 327.

Provenance: Cultivated in Calcutta Botanic Garden from seed from 'interior parts of [North] India'.

Hippocratea indica Willd., Sp. Pl. 1 (1797) 193; Roxb., Pl. Coromandel 2 (1800) 16, t. 130 .

Reissantia indica (Willd.) N. Hallé, Bull. Mus. Hist. Nat. (Paris), sér. 2, 30 (1958) 466. Provenance: Forests, hills, and wild uninhabited places [India].

\section{COMBRETACEAE}

Getonia floribunda Roxb., Pl. Coromandel 1 (1798) 61, t. 87.

Calycopteris floribunda (Roxb.) Poir. in Lam., Encycl., Suppl. 2 (1811) 41 \& Tabl. Encycl. 2 (1793) t. 357.

Provenance: Forests [Circars].

Combretum decandrum Roxb., Pl. Coromandel 1 (1796) 43, t. 59, non Jacq., Enum. Pl. Carib (1760).

Combretum roxburghii Spreng., Syst. Veg. 2 (1825) 331.

Provenance: Forests and mountains [Circars].

Terminalia bellirica (Gaertn.) Roxb., Pl. Coromandel 2 (1805) 54, t. 198 (as 'bellerica').

Myrobalanus bellirica Gaertn., Fruct. Sem. Pl. 2 (1791) 90, t. 97.

Provenance: Circar mountains.

Terminalia chebula Retz., Observ. Bot. 5 (1788) 31; Roxb., Pl. Coromandel 2 (1805) 52, t. 197.

Provenance: Circar mountains.

Terminalia procera Roxb., Pl. Coromandel 3 (1811) 18, t. 224.

Provenance: Cultivated in Calcutta Botanic Garden from the Andaman Islands.

\section{COMMELINACEAE}

Tradescantia axillaris (L.) L., Mant. Pl. 2 (1771) 371; Roxb., Pl. Coromandel 2 (1799) 5, t. 107.

Cyanotis axillaris (L.) Sweet, Hort. Brit. (1827) 430.

Commelina axillaris L., Sp. Pl. (1753) 42.

Provenance: Moist pasture ground, and borders of paddy fields [Circars]. 
Tradescantia tuberosa Roxb., Pl. Coromandel 2 (1799) 5, t. 108.

Cyanotis tuberosa (Roxb.) Schult. \& Schult.f. in Roemer \& Schultes, Syst. Veg. 7 (1830) 1153.

Provenance: Moist valleys.

Tradescantia paniculata Roxb., Pl. Coromandel 2 (1799) 6, t. 109.

Floscopa scandens Lour., Fl. Cochinch. 1 (1790) 193.

Provenance: Moist valleys [Circars].

\section{COMPOSITAE (ASTERACEAE)}

Caesulia axillaris Roxb., Pl. Coromandel 1 (1798) 64, t. 93. Provenance: Moist places.

\section{CONVOLVULACEAE}

Cuscuta reflexa Roxb., Pl. Coromandel 2 (1799) 3, t. 104. Provenance: [Circars].

Erycibe paniculata Roxb., Pl. Coromandel 2 (1802) 31, t. 159.

Provenance: Mountain forests [Circars].

Porana paniculata Roxb., Pl. Coromandel 3 (1815) 31, t. 235.

Poranopsis paniculata (Roxb.) Roberty, Novon 3 (1993) 198.

Provenance: Interior parts of Bengal: upon the ruins of Gour and Rajmahal hills.

\section{CYPERACEAE}

Scirpus tuberosus Roxb., Pl. Coromandel 3 (1815) 25, t. 231 non S. tuberosus Desf., Fl. Atlant. 1 (1798) 50.

Eleocharis dulcis (Burm.f.) Trin. ex Hensch., Vita Rumph. (1833) 186. Andropogon dulce Burm.f., Fl. Ind. (1768) 219.

Provenance: Cultivated in Calcutta Botanic Garden from Canton (China).

\section{DILLENIACEAE}

Dillenia pentagyna Roxb., Pl. Coromandel 1 (1795) 21, t. 20.

Provenance: Valleys far up among the mountains [Circars].

\section{DIPTEROCARPACEAE}

Dipterocarpus turbinatus C.F. Gaertn., Suppl. Carp. 3 (1805) 51, t. 188.1; Roxb., Pl. Coromandel 3 (1811) 10, t. 213.

Provenance: Tipperah, Chittagong, Pegu.

Hopea odorata Roxb., Pl. Coromandel 3 (1811) 7, t. 210.

Provenance: Cultivated near Calcutta from Chittagong. 
Shorea robusta Roxb. ex C.F. Gaertn., Suppl. Carp. 3 (1805) 48, t. 186; Roxb., Pl. Coromandel 3 (1811) 9, t. 212.

Provenance: Northern mountains of India.

Vateria indica L., Sp. Pl. (1753) 515; Roxb., Pl. Coromandel 3 (1820) 86, t. 288.

Provenance: Bidanore country, Malabar.

\section{DRACAENACEAE}

Sansevieria zeylanica auct. non (L.) Willd. (1799); Roxb., Pl. Coromandel 2 (1805) 43, t. 184.

Sansevieria roxburghiana Schult. \& Schult.f., Mant. Syst. Veg. 7 (1829) 357, f. 12 D \& E.

Provenance: In thin jungle [Circars].

\section{EBENACEAE}

Diospyros chloroxylon Roxb., Pl. Coromandel 1 (1795) 38, t. 49.

Provenance: Orixa mountains [?Circars].

Diospyros cordifolia Roxb., Pl. Coromandel 1 (1795) 38, t. 50.

Provenance: Circar mountains.

Ferreola buxifolia (Rottb.) Roxb., Pl. Coromandel 1 (1795) 35, t. 45.

Pisonia buxifolia Rottb., Nye Saml. Kongel. Danske Vidensk. Selsk. Skr. 2 (1783) 536, t. 4 , f. 2.

Ehretia ferrea Willd., Phytographia 1 (1794) 4, t. 2, f. 2.

Diospyros ferrea (Willd.) Bakh., Gard. Bull. Straits Settlem. 7 (1933) 162 \& Bull. Jard. Bot. Buitenzorg III, 15 (1936) 50, var. buxifolia (Rottb.) Bakh., Bull. Jard. Bot. Buitenzorg III, 15 (1936) 57.

Provenance: Mountains [of South India].

Diospyros melanoxylon Roxb., Pl. Coromandel 1 (1795) 36, t. 46.

Provenance: Circar mountains.

Diospyros montana Roxb., Pl. Coromandel 1 (1795) 37, t. 48.

Provenance: Circar mountains.

Embryopteris glutinifera Roxb., Pl. Coromandel 1 (1796) 49, t. 70, nom. illeg.

Diospyros peregrina (Gaertn.) Gürke in Engl. \& Prantl, Nat. Pflanzenfam. IV.1 (Fasc. 69) (1891) 164.

Embryopteris peregrina Gaertn., Fruct. Sem. Pl. 1 (1788) 145, t. 29, f. 2.

Provenance: Circar mountains.

Diospyros sylvatica Roxb., Pl. Coromandel 1 (1795) 37, t. 47.

Provenance: Hilly parts of the Circars. 


\section{ELATINACEAE}

Bergia aquatica Roxb., Pl. Coromandel 2 (1800) 22, t. 142, nom. illeg.

Bergia capensis L., Mant. Pl. (1771) 241.

Provenance: Wet places, or floating on sweet water [Circars].

\section{ERYTHROXYLACEAE}

Erythroxylum monogynum Roxb., Pl. Coromandel 1 (1798) 61, t. 88 (as 'Erythroxylon').

Provenance: Mountains in the Circars.

\section{EUPHORBIACEAE}

Stilago diandra Roxb., Pl. Coromandel 2 (1802) 35, t. 166.

Antidesma acidum Retz., Observ. Bot. 5 (1788) 30.

Provenance: Circar mountains.

Antidesma pubescens Roxb., Pl. Coromandel 2 (1802) 35, t. 167.

Antidesma ghaesembilla Gaertn., Fruct. Sem. Pl. 1 (1788) 189.

Provenance: Circar mountains.

Cluytia montana Roxb., Pl. Coromandel 2 (1802) 38, t. 171.

Bridelia montana (Roxb.) Willd., Sp. Pl. 4 (1805) 978.

Provenance: Interior mountains [Circars].

Cluytia spinosa Roxb., Pl. Coromandel 2 (1802) 38, t. 172.

Bridelia retusa (L.) Spreng. in L., Syst. Veg., ed. 16, 3 (1826) 48.

Cluytia retusa L., Sp. Pl. (1753) 1042.

Provenance: Mountains [Circars].

Cluytia scandens Roxb., Pl. Coromandel 2 (1802) 39, t. 173.

Bridelia scandens (Roxb.) Willd., Sp. Pl. 4 (1805) 979.

Provenance: Banks of rivers and watercourses [Circars].

Cluytia collina Roxb., Pl. Coromandel 2 (1802) 37, t. 169.

Cleistanthus collinus (Roxb.) Benth. ex Hook.f., Fl. Brit. India 5 (1887) 274.

Provenance: Circar mountains.

Cluytia patula Roxb., Pl. Coromandel 2 (1802) 37, t. 170.

Cleistanthus patulus (Roxb.) Müll.Arg. in DC., Prodr. 15 (1866) 505.

Provenance: Moist valleys.

Rottlera tinctoria Roxb., Pl. Coromandel 2 (1802) 36, t. 168.

Mallotus philippensis (Lam.) Müll.Arg., Linnaea 34 (1865) 196.

Croton philippense Lam., Encycl. 2 (1786) 206.

Provenance: Circar mountains. 


\section{FAGACEAE}

Quercus armata Roxb., Pl. Coromandel 3 (1820) 92, t. 296.1.

Castanopsis armata (Roxb.) Spach, Hist. Veg. Phan. 11 (1842) 185.

Provenance: Chittagong, Tipperah, Golparah.

Quercus castanicarpa Roxb., Pl. Coromandel 3 (1820) 93, t. 296.2.

Castanopsis armata (Roxb.) Spach, Hist. Veg. Phan. 11 (1842) 185.

Provenance: Chittagong.

\section{FLACOURTIACEAE}

Flacourtia sepiaria Roxb., Pl. Coromandel 1 (1796) 48, t. 68.

Flacourtia indica (Burm.f.) Merr., Interpr. Herb. Amboin. (1917) 377.

Gmelina indica Burm.f., Fl. Indica (1768) 132, t. 39.5.

Provenance: Woody uncultivated parts of the [Coromandel] coast, both on mountains and lowlands.

Flacourtia inermis Roxb., Pl. Coromandel 3 (1811) 16, t. 222.

Provenance: Cultivated in Calcutta Botanic Garden from the Moluccas.

Flacourtia sapida Roxb., Pl. Coromandel 1 (1796) 49, t. 69.

Flacourtia ramontchi L'Hér., Stirp. (1785) 59, t. 30, 30B.

Provenance: Mountainous parts [Circars].

Gynocardia odorata Roxb., Pl. Coromandel 3 (1820) 95, t. 299.

Provenance: Silhet.

\section{GESNERIACEAE}

Incarvillea parasitica Roxb., Pl. Coromandel 3 (1820) 88, t. 291.

Aeschynanthus parasiticus (Roxb.) Wall., Numer. List (1828) 23, n. 796.

Provenance: Cultivated in Calcutta Botanic Garden from the Garrow hills.

\section{GRAMINEAE (POACEAE)}

Bambos arundinacea Retz., Observ. Bot. 5 (1788) 24; Roxb., Pl. Coromandel 1 (1798) 56, t. 79.

Bambusa bambos (L.) Voss in Vilm., Blumengärtnerei 1 (1896) 1189.

Arundo bambos L., Sp. Pl. (1753) 81.

Provenance: Rich moist soil, such as the banks of rivulets and lakes, among the mountains [South India].

Bambos stricta Roxb., Pl. Coromandel 1 (1798) 58, t. 80.

Dendrocalamus strictus (Roxb.) Nees ex Wight \& Arn. in Wight's Cat. (1834) 107.

Provenance: Drier situations (i.e. than B. arundinacea) [Circars]. 
Rottboellia compressa L.f., Suppl. Pl. (1782) 114; Roxb., Pl. Coromandel 2 (1802) 30, t. 156.

Hemarthria compressa (L.f.) R.Br., Prodr. (1810) 207.

Provenance: Borders of lakes [Circars].

Manisuris myuros L., Mant. Pl. 2 (1771) 300; Roxb., Pl. Coromandel 2 (1799) 10, t. 117 (as 'myurus').

Provenance: Dry, elevated, sandy ground [Circars].

Bambusa baccifera Roxb., Pl. Coromandel 3 (1815) 38, t. 243.

Melocanna baccifera (Roxb.) Kurz, Prelim. Rep. For. Veg. Pegu (1875) App. B 94. Provenance: Chittagong Mountains.

Rottboellia setacea Roxb., Pl. Coromandel 2 (1800) 17, t. 132 (above), nom. illeg.

Microchloa indica (L.f.) P. Beauv., Essai Agrostogr. Atlas (1812) t. 20.

Nardus indica L.f., Suppl. Pl. (1782) 105.

Provenance: On old walls.

Manisuris granularis Naezén, Nov. Gram. Gen. (1779) 40; Roxb., Pl. Coromandel 2 (1799) 11, t. 118.

Mnesithea granularis (L.) de Koning \& Sosef, Blumea 31 (1986) 295.

Cenchrus granularis L., Mant. Pl. App. (1771) 575.

Provenance: Among bushes.

Rottboellia perforata Roxb., Pl. Coromandel 2 (1805) 43, t. 182.

Mnesithea laevis (Retz.) Kunth, Révis. Gramin. 1 (1829) 154.

Rottboellia laevis Retz., Observ. Bot. 3 (1783) 11.

Provenance: Low rich pasture ground [Circars].

Rottboellia corymbosa L.f., Suppl. Pl. (1782) 114; Roxb., Pl. Coromandel 2 (1805) 42, t. 181.

Ophiuros exaltatus (L.) Kuntze, Revis. Gen. P1. 2 (1891) 780.

Aegilops exaltata L., Mant. Pl. (1771) 575.

Provenance: Low, rich, pasture ground [Circars].

Rottboellia thomaea (L.f.) J. König, Naturforscher 23 (1788) 210; Roxb., Pl. Coromandel 2 (1800) 17, t. 132 (below).

Oropetium thomaeum (L.f.) Trin., Fund. Agrost. (1820) 98, t. 3.

Nardus thomaea L.f., Suppl. Pl. (1782) 105.

Provenance: On old walls.

Pommereulla cornucopiae L.f., Diss. Nov. Gram. (1779) 31; Roxb., Pl. Coromandel 2 (1800) 17, t. 131.

Provenance: Dry uncultivated grounds.

Rottboellia exaltata L.f., Suppl. Pl. (1782) 114, non (L.) L.f. (1779); Roxb., Pl. Coromandel 2 (1802) 30,t. 157.

Rottboellia cochinchinensis (Lour.) Clayton, Kew Bull. 35 (1981) 817.

Stegosia cochinchinensis Lour., Fl. Cochinch. 1 (1790) 51.

Provenance: Mountains [Circars]. 
Saccharum sinense Roxb., Pl. Coromandel 3 (1815) 26, t. 232.

Provenance: Cultivated in Calcutta Botanic Garden from China.

Panicum squarrosum Retz., Observ. Bot. 4 (1796) 15; Roxb., Pl. Coromandel 3 (1811) 4, t. 206.

Trachys muricata (L.) Pers. ex Trin., Mém. Acad. Imp. Sci. St.-Pétersbourg, sér. 6, Sci. Math. 3 (1834) 189.

Cenchrus muricatus L., Mant. Pl. (1771) 302.

Provenance: Dry, sandy ground near the sea, Coast of Coromandel.

\section{GUTTIFERAE}

Xanthochymus dulcis Roxb., Pl. Coromandel 3 (1820) 66, t. 270.

Garcinia dulcis (Roxb.) Kurz, J. Asiat. Soc. Bengal 43 (1874) 88, p.p. \& For. Fl. Brit. Burma 1 (1877) 92, p.p.

Provenance: Cultivated in Calcutta Botanic Garden from the Moluccas.

Garcinia cambogia (Gaertn.) Desr. in Lam., Encycl. 3 (1792) 701; Roxb., Pl. Coromandel 3 (1820) 94, t. 298.

Garcinia gummi-gutta (L.) Robson, Brittonia 20 (1968) 103.

Cambogia gummi-gutta L., Gen. Pl., ed. 5 (1754) 522.

Provenance: Travancore.

Xanthochymus pictorius Roxb., Pl. Coromandel 2 (1805) 51, t. 196.

Garcinia xanthochymus Hook.f., Fl. Brit. India 1 (1874) 269.

Provenance: Circar mountains.

\section{HERNANDIACEAE}

Gyrocarpus jacquini Gaertn., Fruct. Sem. Pl. 2 (1791) 92, t. 97.3, nom. illeg.; Roxb., Pl. Coromandel 1 (1795) 1, t. 1.

Gyrocarpus asiaticus Willd., Sp. Pl. 4 (1806) 982 - see Verdc., Kew Bull. 21 (1967) 254.

Provenance: Mountainous parts of the [Coromandel] Coast.

\section{HYDROCHARITACEAE}

Vallisneria octandra Roxb., Pl. Coromandel 2 (1802) 34, t. 165.

Blyxa octandra (Roxb.) Planch. ex Thwaites, Enum. Pl. Zeyl. (1864) 332.

Provenance: Stagnant, shallow, sweet water.

Serpicula verticillata L.f., Suppl. Pl. (1782) 416; Roxb., Pl. Coromandel 2 (1802) 33, t. 164.

Hydrilla verticillata (L.f.) Royle, Ill. Bot. Himal. (1839) t. 376.

Provenance: Clear standing sweet water [Circars]. 
Damasonium indicum Willd., Sp. Pl. 2 (1799) 276; Roxb., Pl. Coromandel 2 (1805) 45, t. 185.

Ottelia alismoides (L.) Pers., Syn. Pl. 1 (1805) 400.

Stratioes alismoides L., Sp. Pl. (1753) 535.

Provenance: Shallow sweet water [Circars].

\section{HYPOXIDACEAE}

Curculigo orchioides Gaertn., Fruct. Sem. Pl. 1 (1788) 63, t. 16, f. 11; Roxb., Pl. Coromandel 1 (1795) 14, t. 13.

Provenance: Uncultivated places about Samulcotah.

\section{LABIATAE (LAMIACEAE)}

Colebrookea ternifolia Roxb., Pl. Coromandel 3 (1815) 40, t. 245.

Colebrookea oppositifolia Sm., Exot. Bot. 2 (1805) 111, t. 115.

Provenance: Cultivated in Calcutta Botanic Garden from Mysore.

\section{LAURACEAE}

Laurus involucrata Roxb., Pl. Coromandel 2 (1805) 46, t. 187.

Litsea glutinosa (Lour.) C.B. Rob., Philipp. J. Sci., Bot. 6 (1911) 321.

Sebifera glutinosa Lour., Fl. Cochinch. (1790) 638.

Provenance: Circar mountains.

Tetranthera apetala Roxb., Pl. Coromandel 2 (1800) 25, t. 147.

Litsea glutinosa (Lour.) C.B. Rob., Philipp. J. Sci., Bot. 6 (1911) 321.

Sebifera glutinosa Lour., Fl. Cochinch. (1790) 638.

Provenance: Circar mountains.

Tetranthera monopetala Roxb., Pl. Coromandel 2 (1800) 26, t. 148.

Litsea monopetala (Roxb.) Pers., Syn. Pl. 2 (1806) 4.

Provenance: Valleys [Circars].

\section{LECYTHIDACEAE}

Careya arborea Roxb., Pl. Coromandel 3 (1811) 14, t. 218.

Provenance: Circar mountains and other mountainous parts of India.

Careya herbacea Roxb., Pl. Coromandel 3 (1811) 13, t. 217.

Provenance: Cultivated in Calcutta Botanic Garden from Rungpore district of Bengal. 


\section{LEGUMINOSAE - CAESALPINIOIDEAE}

Bauhinia anguina Roxb., Pl. Coromandel 3 (1820) 82, t. 285.

Bauhinia scandens L., Sp. Pl. (1753) 374 var. horsfieldii (Miq.) Ohashi in Hara, Enum. Fl. Pl. Nepal 2 (1979) 108.

Lasiobema horsfieldii Miq., Fl. Ind. Bat. 1, 1 (1855) 71.

Provenance: Silhet, Chittagong.

Caesalpinia sappan L., Sp. Pl. (1753) 381; Roxb., Pl. Coromandel 1 (1795) 17, t. 16. Provenance: Circar mountains.

Cynometra polyandra Roxb., Pl. Coromandel 3 (1820) 83, t. 286.

Provenance: Silhet.

Hardwickia binata Roxb., Pl. Coromandel 3 (1811) 6, t. 209.

Provenance: Coromandel mountains.

\section{LEGUMINOSAE - MIMOSOIDEAE}

Mimosa catechu L.f., Suppl. Pl. (1782) 39; Roxb., Pl. Coromandel 2 (1802) 40, t. 175. Acacia catechu (L.f.) Willd., Sp. Pl. 4 (1806) 1079.

Provenance: Mountainous parts of the [Coromandel] coast.

Mimosa sundra Roxb., Pl. Coromandel 3 (1811) 19, t. 225.

Acacia chundra (Rottler) Willd., Sp. Pl. 4 (1806) 1078.

Mimosa chundra Rottler, Ges. Naturf. Freunde Berlin Neue Schriften 4 (1803) 207.

Provenance: Coromandel mountains.

Mimosa eburnea L.f., Suppl. Pl. (1782) 437; Roxb., Pl. Coromandel 2 (1805) 54, t. 199.

Acacia eburnea (L.f.) Willd., Sp. Pl. 4 (1806) 1081.

Provenance: Forests [Circars].

Mimosa leucophloea Roxb., Pl. Coromandel 2 (1800) 27, t. 150.

Acacia leucophloea (Roxb.) Willd., Sp. Pl. 4 (1806) 1083.

Provenance: Dry mountainous districts [Circars].

Mimosa arabica Lam., Encycl. 1 (1783) 19; Roxb., Pl. Coromandel 2 (1800) 26, t. 149.

Acacia nilotica (L.) Delile, Descr. Egypte, Hist. Nat. Ill. (1813) 79.

Mimosa nilotica L., Sp. Pl. (1753) 521.

Provenance: Every part of India.

Mimosa amara Roxb., Pl. Coromandel 2 (1799) 13, t. 122.

Albizia amara (Roxb.) Boivin, Encycl. XIXme Siècle 2 (1838) 34.

Provenance: Mountains [Circars]. 
Mimosa odoratissima L.f., Suppl. Pl. (1782) 437; Roxb., Pl. Coromandel 2 (1799) 12 , t. 120.

Albizia odoratissima (L.f.) Benth., London J. Bot. 3 (1844) 88.

Provenance: Mountainous parts of the coast [Circars].

Mimosa procera Roxb., Pl. Coromandel 2 (1799) 12, t. 121.

Albizia procera (Roxb.) Benth., London J. Bot. 3 (1844) 89.

Provenance: Mountainous parts of the [Coromandel] coast.

Mimosa cinerea L., Sp. Pl. (1753) 520; Roxb., Pl. Coromandel 2 (1802) 39, t. 174.

Dichrostachys cinerea (L.) Wight \& Arn., Prodr. Fl. Ind. Orient. (1834) 271.

Provenance: Forests and low barren lands [Circars].

Mimosa octandra Roxb., Pl. Coromandel 2 (1805) 55, t. 200.

Mimosa intsia L., Sp. Pl. (1753) 522.

Provenance: Thickets, banks of aqueducts and other moist places [Circars].

Mimosa natans Vahl, Symb. Bot. 3 (1794) 102; Roxb., Pl. Coromandel 2 (1799) 11, t. 119.

Neptunia oleracea Lour., Fl. Cochinch. (1790) 654.

Provenance: Pools and lakes of fresh water [Circars].

Mimosa dulcis Roxb., Pl. Coromandel 1 (1798) 67, t. 99.

Pithecellobium dulce (Roxb.) Benth., London J. Bot. 3 (1844) 199.

Provenance: Coromandel coast, introduced from the Philippines.

Prosopis spicigera L., Mant. Pl. (1767) 68; Roxb., Pl. Coromandel 1 (1796) 45, t. 63.

Prosopis cineraria (L.) Druce, Rep. Bot. Exch. Club 3 (1914) 422.

Mimosa cineraria L., Sp. Pl. (1753) 517.

Provenance: Most parts of the coast [i.e. of South East India].

Mimosa xylocarpa Roxb., Pl. Coromandel 1 (1798) 68, t. 100.

Xylia xylocarpa (Roxb.) Taub., Bot. Centralbl. 47 (1891) 395.

Provenance: Circar mountains.

\section{LEGUMINOSAE - PAPILIONOIDEAE (FABOIDEAE)}

Hedysarum bupleurifolium L., Sp. Pl. (1753) 745; Roxb., Pl. Coromandel 2 (1805) 50, t. 194.

Alysicarpus bupleurifolius (L.) DC., Prodr. 2 (1825) 352.

Provenance: Generally [in India] among long grass, on dry ground.

Butea frondosa J. König ex Roxb., Asiat. Res. 3 (1792) 469; Roxb., Pl. Coromandel 1 (1795) 21, t. 21.

Butea monosperma (Lam.) Taub., in Engl. \& Prantl, Nat. Pflanzenfam. 3.3, 104 (1894) 366.

Erythrina monosperma Lam., Encycl. 1 (1785) 391.

Provenance: Lowlands of [Coromandel] coast and mountains. 
Butea superba Roxb., Pl. Coromandel 1 (1795) 23, t. 22.

Provenance: Mountains [Circars].

Crotalaria juncea L., Sp. Pl. (1753) 714; Roxb., Pl. Coromandel 2 (1805) 49, t. 193. Provenance: Cultivated [Circars].

Dalbergia latifolia Roxb., Pl. Coromandel 2 (1799) 7, t. 113.

Provenance: Mountains [Circars].

Dalbergia paniculata Roxb., Pl. Coromandel 2 (1799) 8, t. 114.

Provenance: Circar mountains.

Dalbergia rubiginosa Roxb., Pl. Coromandel 2 (1799) 9, t. 115.

Provenance: Mountains [Circars].

Dalbergia volubilis Roxb., Pl. Coromandel 2 (1805) 48, t. 191.

Provenance: Mountainous countries on the coast [Circars].

Podalyria bracteata Roxb., Pl. Coromandel 3 (1820) 55, t. 259.

Dalhousiea bracteata (Roxb.) Graham in Wall., Numer. List (1831-1832) 185,

n. 5339.

Provenance: Silhet.

Dalbergia scandens Roxb., Pl. Coromandel 2 (1805) 49, t. 192.

Derris scandens (Roxb.) Benth., J. Proc. Linn. Soc., Bot. 4, Suppl. (1860) 103.

Provenance: Common [Circars].

Erythrina arborescens Roxb., Pl. Coromandel 3 (1811) 14, t. 219.

Provenance: Cultivated in Calcutta Botanic Garden from Nepal.

Erythrina resupinata Roxb., Pl. Coromandel 3 (1811) 15, t. 220.

Provenance: 'Hindoostan' [i.e. North India].

Flemingia semialata Roxb., Pl. Coromandel 3 (1815) 45, t. 249.

Provenance: Cultivated in Calcutta Botanic Garden from Nepal.

Flemingia stricta Roxb., Pl. Coromandel 3 (1815) 44, t. 248.

Provenance: Mountains in northern parts of Coromandel coast.

Indigofera linifolia (L.f.) Retz., Observ. Bot. 4 (1786-1787) 29; Roxb., Pl. Coromandel 2 (1805) 50, t. 195.

Hedysarum linifolium L.f., Suppl. Pl. (1782) 331.

Provenance: Dry, sandy, pasture ground [Coromandel coast].

Inocarpus edulis J.R. Forst. \& G. Forst., Char. Gen. (1776) 66; Roxb., Pl. Coromandel 3 (1820) 59, t. 263.

Inocarpus fagifer (Parkinson) Fosberg, J. Wash. Acad. Sci. 31, 3 (1941) 95.

Aniotum fagiferum Parkinson, J. Voyage Endeavour (1773) 39.

Provenance: Cultivated in Calcutta Botanic Garden from the Moluccas. 
Cylista scariosa Roxb., Pl. Coromandel 1 (1798) 64, t. 92.

Paracalyx scariosus (Roxb.) Ali, Univ. Stud. (Karachi) 5, 3 (1968) 95.

Provenance: Circar mountains.

Pterocarpus marsupium Roxb., Pl. Coromandel 2 (1799) 9, t. 116.

Provenance: Circar mountains.

Cylista tomentosa Roxb., Pl. Coromandel 3 (1811) 16, t. 221.

Rhynchosia hirta (Andrews) Meikle \& Verdc., Taxon 16 (1967) 462.

Dolichos hirtus Andrews, Bot. Repos. 7 (1807) t. 446.

Provenance: Cultivated in Calcutta Botanic Garden from Mysore.

\section{LENTIBULARIACEAE}

Utricularia stellaris L.f., Suppl. Pl. (1782) 86; Roxb., Pl. Coromandel 2 (1805) 42, t. 180.

Provenance: Sweet water [Circars].

\section{LOGANIACEAE}

Strychnos nux-vomica L., Sp. Pl. (1753) 189; Roxb., Pl. Coromandel 1 (1795) 8, t. 4. Provenance: Almost every part of the [Coromandel] coast.

Strychnos potatorum L.f., Suppl. Pl. (1782) 148; Roxb., Pl. Coromandel 1 (1795) 9, t. 5.

Provenance: Mountains and woods of great extent [South India].

\section{LORANTHACEAE}

Loranthus bicolor Roxb., Pl. Coromandel 2 (1800) 20, t. 139.

Dendrophthoe falcata (L.f.) Ettingsh., Akad. Wiss. Wien, Math.-Naturwiss. Kl., Denkschr. 32 (1872) 68, t. 13, f. 14, 15.

Loranthus falcatus L.f., Suppl. Pl. (1782) 211.

Provenance: Upon the branches of various trees [Circars].

Loranthus scurrula (L.) L., Sp. Pl., ed. 2 (1762) 472; Roxb., Pl. Coromandel 2 (1800) 21, t. 140.

Scurrula parasitica L., Sp. Pl. (1753) 110.

Provenance: [Circars].

\section{LYTHRACEAE}

Ammannia octandra L.f., Suppl. Pl. (1782) 127; Roxb., Pl. Coromandel 2 (1800) 18, t. 133.

Provenance: Moist places.

Lagerstroemia parviflora Roxb., Pl. Coromandel 1 (1796) 47, t. 66.

Provenance: Circar mountains. 
Lagerstroemia reginae Roxb., Pl. Coromandel 1 (1796) 46, t. 65, nom. illeg.

Lagerstroemia speciosa (L.) Pers., Syn. Pl. 2 (1806) 72.

Munchausia speciosa L., Mant. Pl. 2 (1771) 243.

Provenance: Mountains of northern part of Circars.

Grislea tomentosa Roxb., Pl. Coromandel 1 (1795) 29, t. 31, nom. illeg.

Woodfordia fruticosa (L.) Kurz, J. Asiat. Soc. Bengal 40 (1871) 56.

Lythrum fruticosum L., Sp. Pl. (1762) 641.

Provenance: Hills and valleys of northern provinces [of the Circars].

\section{MAGNOLIACEAE}

Magnolia pterocarpa Roxb., Pl. Coromandel 3 (1820) 62, t. 266.

Provenance: Silhet, Chittagong.

\section{MALPIGHIACEAE}

Triopteris indica Willd., Sp. Pl. 2 (1799) 744; Roxb., Pl. Coromandel 2 (1802) 32, t. 160.

Aspidopterys indica (Willd.) W. Theob. in Mason, Burma 2 (1883) 599.

Provenance: Forests among the mountains [Circars].

Gaertnera racemosa (Cav.) Roxb., Pl. Coromandel 1 (1795) 19, t. 18.

Molina racemosa Cav., Diss. 435 (1790) t. 263.

Note - Roxburgh's misinterpreted Cavanilles' name, which applies to a South American species. The combination is legitimate, but Roxburgh was wrong to use it as he also cites Banisteria benghalensis L., Sp. Pl. (1753) 427 as a synonym. The correct name for Roxburgh's plant is:

Hiptage benghalensis (L.) Kurz, J. Asiat. Soc. Bengal, Pt. 2, Nat. Hist. 43 (1874) 136.

Provenance: Circar Mountains.

\section{MALVACEAE}

Gossypium herbaceum L., Sp. Pl. (1753) 693; Roxb., Pl. Coromandel 3 (1820) 65, t. 269.

Provenance: The widely cultivated commercial crop plant.

Hibiscus cannabinus L., Syst. Nat., ed. 10 (1759) 1149; Roxb., Pl. Coromandel 2 (1805) 48, t. 190.

Provenance: Cultivated [Circars].

Kydia calycina Roxb., Pl. Coromandel 3 (1811) 12, t. 215.

Provenance: Coromandel and Hindoostan [i.e. northern India].

Kydia fraterna Roxb., Pl. Coromandel 3 (1811) 12, t. 216.

(Generally considered to be synonymous with $K$. calycina).

Provenance: Circar mountains. 


\section{MELASTOMATACEAE}

Memecylon edule Roxb., Pl. Coromandel 1 (1798) 59, t. 82.

Provenance: Every jungle on the [Coromandel] coast.

\section{MELIACEAE}

Amoora cucullata Roxb., Pl. Coromandel 3 (1820) 54, t. 258.

Aglaia cucullata (Roxb.) Pellegr. in Lecomte, Fl. Indo-Chine 1 (1911) 171. Provenance: Ganges delta.

Heynea trijuga Roxb. in Curtis's Bot. Mag. (1815) t. 1738; Roxb., Pl. Coromandel 3 (1820) 56, t. 260.

Provenance: Nepal.

Sandoricum indicum Cav., Diss. 7 (1789) 359, t. 202, 203; Roxb., Pl. Coromandel 3 (1820) 57, t. 261

Sandoricum koetjape (Burm.f.) Merr., Philipp. J. Sci., Bot. 7 (1912) 237.

Melia koetjape Burm.f., Fl. Ind. (1768) 101.

Provenance: Cultivated in Calcutta Botanic Garden from the Moluccas.

Swietenia febrifuga Roxb., Pl. Coromandel 1 (1795) 18, t. 17.

Soymida febrifuga (Roxb.) A. Juss., Mem. Mus. Hist Nat. 19 (1830) 251, t. 11, f. 26.

Provenance: Mountainous part of the Rajahmundry Circar, north of Samulcotah and Peddapore.

Cedrela toona Roxb. ex Rottler, Ges. Naturf. Freunde Berlin Neue Schriften 4 (1803) 198; Roxb., Pl. Coromandel 3 (1815) 33, t. 238.

Toona ciliata M. Roem., Fam. Nat. Syn. Monogr. 1. Hesper. (1846) 139.

Provenance: Bengal.

\section{MELIOSMACEAE}

Millingtonia simplicifolia Roxb., Pl. Coromandel 3 (1820) 50, t. 254.

Meliosma simplicifolia (Roxb.) Walp., Repert. Bot. Syst. 1 (1842) 423.

Provenance: Silhet.

\section{MENYANTHACEAE}

Menyanthes cristata Roxb., Pl. Coromandel 2 (1799) 3, t. 105.

Nymphoides hydrophylla (Lour.) Kuntze, Revis. Gen. Pl. (1891) 429.

Menyanthes hydrophylla Lour., Fl. Cochinch. 1 (1790) 129.

Provenance: Tanks, or pools of fresh water [Circars]. 


\section{MORACEAE}

Artocarpus integrifolius auct. non L.f. (1781); Roxb., Pl. Coromandel 3 (1815) 46, t. 250.

Artocarpus heterophyllus Lam., Encycl. 3 (1789) 209.

Provenance: Cultivated over the warmer parts of Asia.

Ficus comosa Roxb., Pl. Coromandel 2 (1799) 14,t. 125.

Ficus benjamina L. var. nuda (Miq.) Barret, Amer. Midl. Naturalist 45 (1951) 127.

Urostigma nudum Miq., London J. Bot. 6 (1847) 584.

Provenance: Circar mountains.

Ficus oppositifolia Roxb., Pl. Coromandel 2 (1799) 14, t. 124.

Ficus hispida L.f., Suppl. Pl. (1782) 442.

Provenance: Banks of rivulets [Circars].

Ficus glomerata Roxb., Pl. Coromandel 2 (1799) 13, t. 123.

Ficus racemosa L., Sp. Pl. (1753) 1060.

Provenance: About villages, and on the banks of rivers and watercourses [Circars].

\section{MUSACEAE}

Musa glauca Roxb., Pl. Coromandel 3 (1820) 96, t. 300.

Ensete glaucum (Roxb.) Cheesman, Kew Bull. 1947 (1948) 101.

Provenance: Cultivated in Calcutta Botanic Garden from Pegu [Burma].

Musa superba Roxb., Pl. Coromandel 3 (1811) 17, t. 223.

Ensete superbum (Roxb.) Cheesman, Kew Bull. 1947 (1948) 100.

Provenance: Cultivated in Calcutta Botanic Garden from valleys 'in the southern parts of the peninsula of India' (via Madras).

Musa sapientum auct. non L. (1759): Roxb., Pl. Coromandel 3 (1820) 73, t. 275.

Musa balbisiana Colla, Mem. Gen. Musa (1820) 56.

Provenance: Cultivated in Calcutta Botanic Garden from wild seed from Chittagong.

\section{MYRISTICACEAE}

Myristica aromatica Lam., Act. Paris. (1788) 155, t. 5-7; Roxb., Pl. Coromandel 3 (1820) 70, t. 274.

Myristica fragrans Houtt., Nat. Hist. 2, 3 (1774) 333.

Provenance: Cultivated in Calcutta Botanic Garden from the Moluccas.

\section{MYRSINACEAE}

Ardisia solanacea Roxb., Pl. Coromandel 1 (1795) 27, t. 27.

Provenance: Moist places in the valleys among the [Circar] mountains. 


\section{NYMPHAEACEAE}

Euryale ferox Salisb., Ann. Bot. 2 (1805) 74; Roxb., Pl. Coromandel 3 (1815) 39, t. 244.

Provenance: Tipperah and Chittagong.

\section{OCHNACEAE}

Ochna squarrosa auct. non L. (1763): Roxb., Pl. Coromandel 1 (1798) 62, t. 89.

Ochna obtusata DC., Ann. Mus. Natl. Hist. Nat. 17 (1811) 411, t. 1.

Provenance: Circar mountains.

\section{OLACACEAE}

Olax scandens Roxb., Pl. Coromandel 2 (1799) 2, t. 102.

Provenance: Forests [Circars].

\section{OLEACEAE}

Schrebera swietenioides Roxb., Pl. Coromandel 2 (1799) 1, t. 101. Provenance: Mountainous part of the Rajahmundry Circar.

\section{OPILIACEAE}

Cansjera scandens Roxb., Pl. Coromandel 2 (1799) 2, t. 103 (as 'Cansiera').

Cansjera rheedii J.F. Gmel. in L., Syst. Nat., ed. 13, 2, 1 (1791) 280.

Provenance: Circar mountains.

Opilia amentacea Roxb., Pl. Coromandel 2 (1802) 31, t. 158.

Provenance: Circar mountains.

\section{ORCHIDACEAE}

Epidendrum praemorsum Roxb., Pl. Coromandel 1 (1795) 34, t. 43.

Acampe praemorsa (Roxb.) Blatt. \& McCann, J. Bombay Nat. Hist. Soc. 35 (1932) 495.

Provenance: Circar mountains.

Aerides multiflorum Roxb., Pl. Coromandel 3 (1820) 68, t. 271.

Provenance: Garrow hills near Silhet.

Epidendrum pendulum Roxb., Pl. Coromandel 1 (1795) 35, t. 44.

Cymbidium pendulum (Roxb.) Sw., Nova Act. Regiae Soc. Sci. Upsal. 2, 6 (1799) 73.

Provenance: Circar mountains.

Limodorum aphyllum Roxb., Pl. Coromandel 1 (1795) 34, t. 41.

Dendrobium aphyllum (Roxb.) C.E.C. Fisch., Fl. Madras 3 (1928) 1416.

Provenance: Dry rocky hills. 
Limodorum virens Roxb., Pl. Coromandel 1 (1795) 32, t. 38.

Eulophia epidendraea (J. König) Schltr., Die Orchideen (1915) 346.

Serapias epidendraea J. König ex Retz., Observ. Bot. 6 (1791) 65.

Provenance: Dry, uncultivated ground.

Limodorum nutans Roxb., Pl. Coromandel 1 (1795) 33, t. 40.

Geodorum densiflorum (Lam.) Schltr., Spec. Nov. Regni Veg. Beih. 4 (1929) 259.

Limodorum densiflorum Lam., Encycl. 3 (1792) 516.

Provenance: Moist valleys among the hills.

Limodorum recurvum Roxb., Pl. Coromandel 1 (1795) 33, t. 39.

Geodorum recurvum (Roxb.) Alston in Trimen, Handb. Fl. Ceylon 6 (1931) 276.

Provenance: Moist valleys among the hills.

Orchis plantaginea Roxb., Pl. Coromandel 1 (1795) 32, t. 37, non Habenaria plantaginea Lindl. (1835).

Habenaria roxburghii Nicolson, Fl. Hassan Dist. (1976) 834.

Provenance: Moist valleys among the hills.

Epidendrum tessellatum Roxb., Pl. Coromandel 1 (1795) 34, t. 42.

Vanda tessellata (Roxb.) Hook. ex D. Don in Loudon, Hort. Brit. (1830) 372.

Provenance: Circar mountains.

\section{PALMAE (ARECACEAE)}

Areca catechu L., Sp. Pl. (1753) 1189; Roxb., Pl. Coromandel 1 (1796) 54, t. 75.

Provenance: Cultivated over every part of India.

Borassus flabelliformis Murray in L., Syst. Veg., ed. 13 (1774) 827; Roxb., Pl. Coromandel 1 (1796) 50, t. 71, 72 .

Borassus flabellifer L., Sp. Pl. (1753) 1187.

Provenance: [Coromandel] coast.

Cocos nucifera L., Sp. Pl. (1753) 1188; Roxb., Pl. Coromandel 1 (1796) 52, t. 73. Provenance: Sandy soil near the sea [south India].

Corypha taliera Roxb., Pl. Coromandel 3 (1820) 51, t. 255, 256 (as 'Coryphea'). Provenance: Bengal.

Phoenix acaulis Roxb., Pl. Coromandel 3 (1820) 69, t. 273.

Provenance: Cultivated in Calcutta Botanic Garden from Bahar.

Phoenix farinifera Roxb., Pl. Coromandel 1 (1796) 53, t. 74.

Phoenix pusilla Gaertn., Fruct. Sem. Pl. 1 (1788) 24, t. 9.

Provenance: Dry, barren ground, and chiefly found on the sandy lands at a small distance from the sea [Circars].

Wallichia caryotoides Roxb., Pl. Coromandel 3 (1820) 91, t. 295.

Provenance: Chittagong. 


\section{PANDANACEAE}

Pandanus odoratissimus L.f., Suppl. Pl. (1782) 64; Roxb., Pl. Coromandel 1 (1798) 65, t. 94-96.

Provenance: Warmer parts of Asia (including Coromandel coast).

\section{PASSIFLORACEAE}

Modecca trilobata Roxb., Pl. Coromandel 3 (1820) 93, t. 297.

Adenia trilobata (Roxb.) Engl., Jahrb. 14 (1891) 375.

Provenance: Chittagong.

\section{PONTEDERIACEAE}

Pontederia hastata L., Sp. Pl. (1753) 288; Roxb., Pl. Coromandel 2 (1799) 6, t. 111.

Monochoria hastata (L.) Solms in A.DC., Monogr. Phan. 4 (1883) 523.

Provenance: Marshy places or shallow, standing, fresh water [Circars].

Pontederia vaginalis Burm.f., Fl. Indica (1768) 80; Roxb., Pl. Coromandel 2 (1799) 6, t. 110.

Monochoria vaginalis (Burm.f.) C. Presl, Reliq. Haenk. 1 (1827) 128. Provenance: Marsy places [Circars].

\section{RANUNCULACEAE}

Atragene zeylanica L., Sp. Pl. (1753) 542; Roxb., Pl. Coromandel 2 (1805) 47, t. 188. Naravelia zeylanica (L.) DC., Syst. Nat. 1 (1817) 167.

Provenance: Samulcotah.

\section{RHAMNACEAE}

Gouania tiliifolia auct. non Lam. (1789); Roxb., Pl. Coromandel 1 (1798) 67, t. 98. Gouania leptostachya DC., Prodr. 2 (1825) 40.

Provenance: Circar mountains.

Ventilago maderaspatana Gaertn., Fruct. Sem. Pl. 1 (1788) 223, t. 49, f. 2; Roxb., Pl. Coromandel 1 (1798) 55, t. 76.

Provenance: Forests and other uncultivated places among the mountains [Circars].

\section{RHIZOPHORACEAE}

Carallia lucida Roxb., Pl. Coromandel 3 (1811) 8, t. 211. Provenance: Circar mountains. 


\section{RUBIACEAE}

Gardenia fragrans Roxb., Pl. Coromandel 2 (1800) 19, t. 137.

Benkara malabarica (Lam.) Tirveng., Taxon 32 (1983) 440.

Randia malabarica Lam., Encycl. 3 (1789) 25.

Provenance: Poor soil [Circars].

Canthium parviflorum Lam., Encycl. 1 (1785) 602; Roxb., Pl. Coromandel 1 (1796) 39, t. 51.

Provenance: On the [Coromandel] coast.

Gardenia latifolia Aiton, Hort. Kew. 1 (1789) 294; Roxb., Pl. Coromandel 2 (1800) 18, t. 134.

Provenance: Circars and Carnatic.

Nauclea cordifolia Roxb., Pl. Coromandel 1 (1796) 40, t. 53.

Haldina cordifolia (Roxb.) Ridsdale, Blumea 24 (1979) 361.

Provenance: Mountainous parts of the [Coromandel] coast.

Hydrophylax maritima L.f., Suppl. Pl. (1782) 126; Roxb., Pl. Coromandel 3 (1815) 29, t. 233.

Provenance: Coastal sand hills of Coromandel and Malabar.

Cinchona excelsa Roxb., Pl. Coromandel 2 (1799) 4, t. 106.

Hymenodictyon orixense (Roxb.) Mabb., Taxon 31 (1982) 66.

Cinchonae orixensis Roxb., Bot. Descr. Swietenia (1793) 21 \& Med. Facts Obs. 6 (1795) 152.

Provenance: Circar mountains.

Nauclea parvifolia Roxb., Pl. Coromandel 1 (1796) 40, t. 52.

Mitragyna parvifolia (Roxb.) Korth., Observ. Naucl. Indic. (1839) 19.

Provenance: Almost every part of the [Coromandel] coast, but chiefly among the mountains.

Morinda angustifolia Roxb., Pl. Coromandel 3 (1815) 32, t. 237.

Provenance: Cultivated in Calcutta Botanic Garden from Chittagong.

Nauclea purpurea Roxb., Pl. Coromandel 1 (1796) 41, t. 54.

Neonauclea purpurea (Roxb.) Merr., Interpr. Rumph. (1917) 483.

Provenance: Circar mountains.

Oldenlandia umbellata L., Sp. Pl. (1753) 119; Roxb., Pl. Coromandel 1 (1795) 2, t. 3.

Provenance: Dry sandy ground near the sea [south east India].

Gardenia dumetorum Retz., Observ. Bot. 2 (1781) 14; Roxb., Pl. Coromandel 2 (1800) 19, t. 136.

Randia dumetorum (Retz.) Poir. in Lam., Encycl. Suppl. 2 (1812) 829 \& Tabl. Encycl. (1791) t. 156.

Provenance: On the [Coromandel] coast. 
Spermadictyon suaveolens Roxb., Pl. Coromandel 3 (1815) 32, t. 236.

Provenance: Cultivated in Calcutta Botanic Garden from the Rajmahal hills (Bihar).

Gardenia uliginosa Retz., Observ. Bot. 2 (1781) 14; Roxb., Pl. Coromandel 2 (1800) 19, t. 135.

Tamilnadia uliginosa (Retz.) Tirveng. \& Sastre, Maurit. Inst. Bull. 8, 4 (1979) 85.

Provenance: Banks of rivers and lowlands [Circars].

\section{RUTACEAE}

Aegle marmelos (L.) Corrêa, Trans. Linn. Soc. London 5 (1800) 223; Roxb., Pl. Coromandel 2 (1800) 23, t. 143.

Crateva marmelos L., Sp. Pl. (1753) 444.

Provenance: Mountainous parts of the coast [Circars].

Limonia monophylla L., Mant. Pl. (1775) 237; Roxb., Pl. Coromandel 1 (1798) 59, t. 83 .

Atalantia monophylla (L.) Corrêa, Ann. Mus. Natl. Hist. Nat. 6 (1805) 383.

Provenance: Forests on the coast [Circars].

Swietenia chloroxylon Roxb., Pl. Coromandel 1 (1796) 46, t. 64.

Chloroxylon swietenia DC., Prodr. 1 (1824) 625.

Provenance: Circar mountains.

Limonia pentaphylla auct. non Retz. (1788); Roxb., Pl. Coromandel 1 (1798) 60, t. 84. Glycosmis mauritiana (Lam.) Tanaka, Bot. Not. 28 (1928) 159, Bull. Soc. Bot. France 75 (1928) 708.

Limonia mauritiana Lam., Encycl. 3 (1788, 1792) 517.

Provenance: Most uncultivated lands [Circars].

Limonia arborea Roxb., Pl. Coromandel 1 (1798) 60, t. 85.

Glycosmis pentaphylla (Retz.) DC., Prodr. 1 (1824) 538 (nomen solum).

Limonia pentaphylla Retz., Observ. Bot. 5 (1788) 24.

Provenance: Circar mountains.

Feronia elephantum Corrêa, Trans. Linn. Soc. London 5 (1800) 225; Roxb., Pl. Coromandel 2 (1800) 21, t. 141.

Limonia acidissima L., Sp. Pl., ed. 2 (1762) 554.

Provenance: Most woods and mountainous parts of India.

Bergera koenigii L., Mant. Pl. 1 (1771) 563; Roxb., Pl. Coromandel 2 (1799) 7, t. 112.

Murraya koenigii (L.) Spreng. in L., Syst. Veg., ed. 16, 2 (1826) 315.

Provenance: Circar mountains; also cultivated.

Limonia crenulata Roxb., Pl. Coromandel 1 (1798) 60, t. 86.

Naringi crenulata (Roxb.) Nicolson in Saldanha \& Nicolson, Fl. Hassan Dist. (1976) 387.

Provenance: Lowlands near the coast and mountains [Circars]. 


\section{SALICACEAE}

Salix tetrasperma Roxb., Pl. Coromandel 1 (1798) 66, t. 97.

Provenance: Banks of rivulets and moist places far among the mountains.

\section{SALVADORACEAE}

Salvadora persica L., Sp. Pl. (1753) 122; Roxb., Pl. Coromandel 1 (1795) 26, t. 26.

Provenance: Most parts of the Circars.

\section{SANTALACEAE}

Sirium myrtifolium auct. non L. (1771); Roxb., Pl. Coromandel 1 (1795) 2, t. 2.

Santalum album L., Sp. Pl. (1753) 349.

Provenance: Many parts of India (including Circar Mountains).

\section{SAPINDACEAE}

Ornitrophe serrata Roxb., Pl. Coromandel 1 (1796) 44, t. 61.

Allophylus serratus (Roxb.) Kurz, J. Asiat. Soc. Bengal, Pt. 2, Nat. Hist. 44 (1876) 185.

Provenance: On the coast and among the mountains [Circars].

Sapindus rubiginosa Roxb., Pl. Coromandel 1 (1796) 44, t. 62.

Lepisanthes rubiginosa (Roxb.) Leenh., Blumea 17 (1969) 82.

Provenance: Circar mountains.

Molinaea canescens Roxb., Pl. Coromandel 1 (1796) 43, t. 60.

Lepisanthes tetraphylla (Vahl) Radlk., Sitzungsber. Math.-Phys. Cl. Königl. Bayer.

Akad. Wiss. München 8 (1878) 276.

Sapindus tetraphylla Vahl, Symb. Bot. 3 (1794) 54.

Provenance: Circar mountains.

\section{SAPOTACEAE}

Bassia latifolia Roxb., Pl. Coromandel 1 (1795) 20, t. 19.

Madhuca indica J.F. Gmel., Syst. Nat. 2, 1 (1791) 799.

Provenance: Mountains parts of the [Coromandel] coast.

Mimusops hexandra Roxb., Pl. Coromandel 1 (1795) 16, t. 15.

Manilkara hexandra (Roxb.) Dubard, Ann. Inst. Bot.-Geol. Colon. Marseille, sér. 3, 23 (1915) 9, f. 2.

Provenance: Mountainous, uncultivated parts of the Circars.

Mimusops elengi L., Sp. Pl. (1753) 349; Roxb., Pl. Coromandel 1 (1795) 15, t. 14.

Provenance: Mountains in the Rajahmundry Circar. 
Sideroxylon tomentosum Roxb., Pl. Coromandel 1 (1795) 28, t. 28.

Xantolis tomentosa (Roxb.) Raf., Sylva Tellur. (1838) 36.

Provenance: Mountains.

\section{SCROPHULARIACEAE}

Orobanche acaulis Roxb., Pl. Coromandel 3 (1820) 89, t. 292.

Aeginetia acaulis (Roxb.) Walp., Repert. Bot. Syst. 3 (1844) 481.

Provenance: Calcutta Botanic Garden as root parasite on sugar cane.

Aeginetia indica L., Sp. Pl. (1753) 632; Roxb., Pl. Coromandel 1 (1798) 63, t. 91. Provenance: Circar mountains.

Gratiola monnieri (L.) L., Syst. Nat., ed. 10 (June 1759) 851 \& Amoen. Acad. 4 (Nov. 1759) 306; Roxb., Pl. Coromandel 2 (1805) 41, t. 178 (as 'monnieria').

Bacopa monnieri (L.) Pennell, Proc. Acad. Nat. Sci. Philadelphia 98 (1946) 94.

Lysimachia monnieri L., Cent. Pl. 2 (1756) 9.

Provenance: Moist places [Circars].

Gratiola juncea Roxb., Pl. Coromandel 2 (1800) 16, t. 129.

Dopatrium junceum (Roxb.) Buch.-Ham. ex Benth., Scroph. Ind. (1835) 31.

Provenance: Wet situations.

Gratiola hyssopioides auct. non L. (1771); Roxb., Pl. Coromandel 2 (1800) 16, t. 128.

Dopatrium lobelioides (Retz.) Benth., Scroph. Ind. (1835) 31.

Gratiola lobelioides Retz., Observ. Bot. 4 (1786) 7.

Provenance: None given.

Cyrilla aquatica Roxb., Pl. Coromandel 2 (1805) 47, t. 189.

Limnophila indica (L.) Druce, Rep. Bot. Exch. Club. 3 (1914) 420.

Hottonia indica L., Syst. Nat., ed. 10 (1759) 919.

Provenance: Borders of pools [Circars].

Gratiola grandiflora Retz., Observ. Bot. 4 (1786-1787) 8; Roxb., Pl. Coromandel 2 (1805) 42, t. 179.

Lindernia antipoda (L.) Alston in Trimen, Fl. Ceylon 6, Suppl. (1931) 214.

Ruellia antipoda L., Sp. Pl. (1753) 635.

Provenance: Moist places.

Gratiola veronicifolia Retz., Observ. Bot. 4 (1786-1787) 8; Roxb., Pl. Coromandel 2 (1802) 29, t. 154.

Lindernia antipoda (L.) Alston in Trimen, Fl. Ceylon 6, Suppl. (1931) 214.

Ruellia antipoda L., Sp. Pl. (1753) 635.

Provenance: Moist places.

Gratiola lucida Vahl, Enum. Pl. 1 (1804) 95; Roxb., Pl. Coromandel 3 (1811) 2, t. 202.

Lindernia crustacea (L.) F. Muell., Syst. Census Austral. Pl. (1882) 97.

Capraria crustacea L., Mant. Pl. 1 (1767) 87.

Provenance: Moist places [all] over India. 
Gratiola oppositifolia Retz., Observ. Bot. 4 (1786-1787) 8; Roxb., Pl. Coromandel 2 (1802) 30, t. 155.

Lindernia oppositifolia (Retz.) Mukh., J. Indian Bot. Soc. 24 (1945) 134.

Provenance: Moist pasture ground.

Gratiola parviflora Roxb., Pl. Coromandel 3 (1811) 3, t. 203.

Lindernia parviflora (Roxb.) Haines, Bot. Bihar Orissa 4 (1922) 635.

Provenance: Low, moist places [all] over India.

Gratiola rotundifolia L., Mant. Pl. 2 (1771) 174; Roxb., Pl. Coromandel 3 (1811) 3 , t. 204.

Lindernia rotundifolia (L.) Alston in Trimen, Fl. Ceylon 6, Suppl. (1931) 214. Provenance: None given.

Gerardia delphinifolia L., Cent. Pl. 2 (1756) 21; Roxb., Pl. Coromandel 1 (1798) 62, t. 90 .

Sopubia delphinifolia (L.) G. Don, Gen. Hist. 4 (1837) 560.

Provenance: Mountainous part of the country.

Stemodia viscosa Roxb., Pl. Coromandel 2 (1802) 33, t. 163.

Provenance: Dry paddy fields [Circars].

Torenia cordifolia Roxb., Pl. Coromandel 2 (1802) 32, t. 161.

Provenance: Moist pasture lands about Samulcotah.

\section{SIMAROUBACEAE}

Ailanthus excelsa Roxb., Pl. Coromandel 1 (1795) 24, t. 23.

Provenance: Circars.

\section{STAPHYLEACEAE}

Darlympelea pomifera Roxb., Pl. Coromandel 3 (1820) 76, t. 279.

Turpinia pomifera (Roxb.) DC., Prodr. 2 (1825) 3.

Provenance: Silhet.

\section{STERCULIACEAE}

Byttneria herbacea Roxb., Pl. Coromandel 1 (1795) 28, t. 29 (as 'Buttneria'). Provenance: Tops of the mountains.

Sterculia colorata Roxb., Pl. Coromandel 1 (1795) 26, t. 25.

Firmiana colorata (Roxb.) R.Br. in Benn. \& R.Br., Pl. Jav. Rar. (1844) 235.

Provenance: Mountainous parts of the Rajahmundry Circar.

Sterculia alata Roxb., Pl. Coromandel 3 (1820) 84, t. 287.

Pterygota alata (Roxb.) R.Br. in Benn. \& R.Br., Pl. Jav. Rar. (1844) 234.

Provenance: Cultivated in Calcutta Botanic Garden from East Bengal (Chittagong and Silhet). 
Sterculia urens Roxb., Pl. Coromandel 1 (1795) 25, t. 24.

Provenance: Mountainous countries on the coast [Circars].

STEMONACEAE

Roxburghia Roxb., Pl. Coromandel 1 (1795) 29, t. 32.

Stemona Lour., Fl. Cochinch. (1790) 404.

Roxburghia gloriosoides Roxb., Pl. Coromandel 1 (1795) 29, t. 32.

Stemona tuberosa Lour., Fl. Cochinch. (1790) 404.

Provenance: Moist valleys among the mountains [Circars].

Note - Various attempts to spare Roxburgh's posthumous blushes over the authorship of these names have been made. In Index Kewensis the genus is attributed to '[Banks in] Roxb.' and Index Nominum Genericorum, more unaccountably, gives 'W. Jones ex Roxb.'. It is possible that J.G. König was the first to suggest them, as Roxburgh (Fl. Ind., ed. 2, 2 (1832) 236) himself paints a touching scene of König examining the plant on his death-bed and asking Roxburgh to describe it. In the same work Roxburgh attributes the generic name to Banks (not unreasonably, as it was he who supervised the publication of the Coromandel Plants) and the species name to Willdenow; however, as there is nothing in the protologue to indicate the role of these authors, the names must be attributed to Roxburgh alone. The origin of any role of Sir William Jones in this story is unclear.

\section{TACCACEAE}

Tacca integrifolia Ker Gawl. in Curtis's Bot. Mag. (1812) t. 1488; Roxb., Pl. Coromandel 3 (1820) 53, t. 257.

Provenance: Cultivated in Calcutta Botanic Garden from 'valleys amongst the hills behind Islamabad, Chittagong'.

\section{TILIACEAE}

Berrya ammonilla Roxb., Pl. Coromandel 3 (1820) 60, t. 264 (as 'Berria').

Berrya cordifolia (Willd.) Burrett, Notizbl. Bot. Gart. Berlin-Dahlem 9 (1926) 606. Espera cordifolia Willd., Ges. Naturf. Freunde Berlin Neue Schriften 3 (1801) 449.

Provenance: Ceylon.

Brownlowia elata Roxb., Pl. Coromandel 3 (1820) 61, t. 265.

Provenance: Chittagong.

\section{TRAPACEAE}

Trapa bispinosa Roxb., Pl. Coromandel 3 (1815) 29, t. 234.

Trapa natans L., Sp. Pl. (1753) 120, var. bispinosa (Roxb.) Makino, Inuma SomokuDzusetze, ed. 3, 1 (1907) 137.

Provenance: Bengal and many other parts of India. 


\section{ULMACEAE}

Ulmus integrifolia Roxb., Pl. Coromandel 1 (1798) 56, t. 78.

Holoptelea integrifolia (Roxb.) Planch., Ann. Sci. Nat. Bot., Sér. 3, 10 (1848) 266. Provenance: Circar mountains.

\section{VERBENACEAE}

Congea tomentosa Roxb., Pl. Coromandel 3 (1820) 90, t. 293.

Provenance: Forests of Chittagong.

Gmelina arborea Roxb., Hort. Beng. (1814) 46; Pl. Coromandel 3 (1815) 41, t. 246. Provenance: Various mountainous parts of India.

Gmelina parvifolia Roxb., Pl. Coromandel 2 (1802) 32, t. 162.

Gmelina asiatica L., Sp. Pl. (1753) 626.

Provenance: Forests and uncultivated places on the [Coromandel] coast.

Streptium asperum Roxb., Pl. Coromandel 2 (1800) 25, t. 146.

Priva cordifolia (L.f.) Druce, Rep. Bot. Exch. Club 4 (1917) 641.

Buchnera cordifolia L.f., Suppl. Pl. (1782) 287.

Provenance: Samulcotah.

Symphorema involucratum Roxb., Pl. Coromandel 2 (1805) 46, t. 186.

Provenance: Forests [Circars].

Tectona grandis L.f., Suppl. Pl. (1782) 151; Roxb., Pl. Coromandel 1 (1795) 10, t. 6.

Provenance: Mountainous part of the Malabar and Coromandel coasts, mountains bordering on the banks of the Godavery above Rajahmundry; Pegu [Burma].

\section{XANTHOPHYLLACEAE}

Xanthophyllum flavescens Roxb., Pl. Coromandel 3 (1820) 82, t. 284 (floral parts only).

Provenance: Chittagong.

Xanthophyllum virens Roxb., Pl. Coromandel 3 (1820) 81, t. 284 (habit and floral parts).

Xanthophyllum flavescens Roxb., Pl. Coromandel 3 (1820) 82, t. 284.

Provenance: Silhet.

\section{ZINGIBERACEAE}

Amomum cardamomum auct. non L. (1753); Roxb., Pl. Coromandel 3 (1815) 21, t. 227.

Amomum compactum Sol. ex Maton, Trans. Linn. Soc. London 10 (1811) 251.

Provenance: Cultivated in Calcutta Botanic Garden from Sumatra and the Malay Islands. 
Alpinia costata Roxb., Pl. Coromandel 3 (1820) 48, t. 252.

Amomum costatum (Roxb.) Baker in Hook.f., Fl. Brit. India 6 (1892) 235.

Provenance: Hilly countries in the vicinity of Silhet.

Amomum subulatum Roxb., Pl. Coromandel 3 (1815) 75, t. 227.

Provenance: Cultivated in Calcutta Botanic Garden from 'lower range of the Morungh mountains skirting the plains of Bengal on the north'.

Curcuma montana Roxb., Pl. Coromandel 2 (1802) 28, t. 151.

Provenance: Moist places among the mountains.

Curcuma zerumbet Roxb., Asiat. Res. 11 (1810) 333; Pl. Coromandel 3 (1811) 1, t. 201, nom. illeg.

Curcuma zedoaria (Christm.) Roscoe, Trans. Linn. Soc. London 8 (1807) 354.

Amomum zedoaria Christm. in Christm. \& Panz., Vollst. Pflanzensyst. 5 (1779) 12.

Provenance: Various parts of India (including Bengal).

Alpinia cardamomum (L.) Roxb., Asiat. Res. 11 (1810) 355; Pl. Coromandel 3 (1815) 19, t. 226 (as 'Amomum cardamomum').

Amomum cardamomum L., Sp. Pl. (1753) 1.

Elettaria cardamomum (L.) Maton, Trans. Linn. Soc. London 10 (1811) 254, t. 5. Provenance: Mountainous parts of the coast of Malabar.

Alpinia linguiformis Roxb., Pl. Coromandel 3 (1820) 74, t. 276 \& Fl. Ind., ed. 1, 1 (1820) 73 .

Etlingera linguiformis (Roxb.) R.M. Sm., Notes Roy. Bot. Gard. Edinburgh 43 (1986) 246.

Provenance: Interior parts of Bengal.

Globba orixensis Roxb., Asiat. Res. 11 (1810) 358, t. 8; Pl. Coromandel 3 (1815) 23, t. 229.

Provenance: Moist valleys up amongst the mountains of the Rajahmundry Circar.

Globba pendula Roxb., Asiat. Res. 11 (1810) 359; P1. Coromandel 3 (1815) 22, t. 228.

Provenance: Pulo-Pinang island.

Globba radicalis Roxb., Asiat. Res. 11 (1810) 359; Pl. Coromandel 3 (1815) 24, t. 230.

Provenance: Cultivated in Calcutta Botanic Garden from Chittagong.

Hedychium angustifolium Ker Gawl. in Edwards, Bot. Regist. 2 (1816) t. 157; Roxb., Pl. Coromandel 3 (1820) 47, t. 251 \& Fl. Ind., ed. 1, 1 (1820) 11.

Hedychium coccineum Buch.-Ham. ex Sm. in Rees, Cycl. 17 (1811) 5. Provenance: Chittagong, Silhet.

Kaempferia ovalifolia Roxb., Pl. Coromandel 3 (1820) 75, t. 278 \& Fl. Ind., ed. 1, 1 (1820) 18.

Provenance: Cultivated in Calcutta Botanic Garden from Malacca peninsula.

Note - Identity and correct name uncertain. 
Zingiber ligulatum Roxb., Asiat. Res. 11 (1810) 348; Pl. Coromandel 3 (1820) 49, t. 253.

Provenance: Hindustan [i.e. northern India].

Note - Identity and correct name uncertain.

Amomum roseum Roxb., Pl. Coromandel 2 (1800) 15, t. 126.

Zingiber roseum (Roxb.) Roscoe, Trans. Linn. Soc. London 8 (1807) 348.

Provenance: Moist valleys [Circars].

\section{ACKNOWLEDGEMENTS}

I am indebted to the following for assistance: Dr H.J. Noltie (E) for practical advice; Dr M. Newman (E) for checking the Zingiberaceae; Dr G. Argent (E) for identifying the Musa. Mr R. Desmond for suggesting relevant references; Drs B. Verdcourt, P.S. Green and T.A. Cope (K) and Dr J.R. Edmondson (LIV) for useful suggestions. And to the home team at Trichy: Bhanumathy Thulasiraj for editing, Hema Ramesh for computer support and A. Rajasekaran for preparing the map.

\section{REFERENCES}

Anonymous. 1823. [Review of] Roxburgh's Plants of the Coast of Coromandel. Flora (Regensburg) 7, 30: 465-477; 7, 31: 481-492.

Beddome, R.H. 1874. Icones Plantarum Indiae Orientalis. Van Voorst, London.

Bretschneider, E. 1898. History of European Botanical Researches in China. Sampson Low, Marston \& Co., London.

Burkill, I.H. 1965. Chapters on the history of botany in India. Government of India Press, Delhi.

Desmond, R. 1977. William Roxburgh's Plants of the Coast of Coromandel 1795-1820. Hortulus Aliquando 2: 22-44.

Desmond, R. 1992. The European discovery of the Indian flora. Royal Botanic Gardens \& Oxford University Press, Oxford.

Desmond, R. 2003. Great natural history books and their creators. The British Library, London.

Forman, L.L. 1997. Notes concerning the typification of names of William Roxburgh's species of phanerogams. Kew Bull. 52: 513-534.

Hedge, I.C. \& J.M. Lamond. 1989. Edinburgh's Indian botanical connections and collections. Bull. Bot. Surv. India 29: 274-275.

Hooker, J.D. (ed.). 1872-1897. The Flora of British India. 7 vols. Reeve \& Co., London.

King, G. 1895. A brief memoir of William Roxburgh. Ann. Roy. Bot. Gard. (Calcutta) 5: 1-9.

Matthew, K.M. 1993. Notes on an important botanical trip (1799-1800) of J.P. Rottler on the Coromandel Coast (India) with a translation of his original text, explanatory notes and a map. Bot. J. Linn. Soc. 113: 351-388.

Merrill, E.D. 1952. A partial list of present locations of authentically named William Roxburgh's phanerogams. (Unpublished typescript, copy in library of Royal Botanic Gardens, Kew, also in L, Fl. Males. Library.)

Morton, C.V. 1974. William Roxburgh's fern types. Contrib. US Natl. Herb. 38: 283-396.

Nayar, M.P. \& A.P. Das. 1984. Glimpses of William Roxburgh through his unpublished letters and his interest in Indian economic botany. J. Econ. Taxon. Bot. 5: 1159-1167.

Nayar, M.P. \& A.P. Das. 1985. William Roxburgh (1751-1815) \& Nathaniel Wallich (1786-1854). Bull. Bot. Surv. India 25: 347-365.

Robinson, T.J. 2003. William Roxburgh (1751-1815), the founding father of Indian botany. Unpublished $\mathrm{PhD}$ dissertation, University of Edinburgh.

Roxburgh, W. 1795-1820. Plants of the coast of Coromandel. 3 vols. Bulmer \& Co., London. Reprinted by Bishen Singh Mahendra Pal Singh, Dehra Dun, 1981.

Roxburgh, W. 1814. Hortus Bengalensis, W. Carey (ed.). Mission Press, Serampore. 
Roxburgh, W. 1820-1824. Flora Indica. 2 vols., W. Carey \& N. Wallich (eds.). Mission Press, Serampore.

Roxburgh, W. 1832. Flora Indica. 3 vols, W. Carey (ed.). Serampore.

Sanjappa, M., Thothathri, K. \& A.R. Das. 1993. Roxburgh's Flora Indica drawings at Calcutta. Bull. Bot. Surv. India 33: 1-232.

Sealy, J.R. 1956. The Roxburgh Flora Indica drawings at Kew. Kew Bull. 11: 297-399.

Sealy, J.R. 1975. William Roxburgh's collection of paintings of Indian plants. Endeavour 34, 122: 84-89.

Stafleu, F.A. \& R.S. Cowan. 1983. Taxonomic literature. Vol. IV: P-Sak., ed. 2. Bohn, Scheltema \& Holkema, Utrecht/Antwerpen.

Thornton, E. 1886. A gazetteer of the territories under the Government of the Viceroy of India. Allen \& Co., London.

Wight, R. 1838-1853. Icones Plantarum Indiae Orientalis. 6 vols. Pharoah, Madras.

Wood, D. 1969. Roxburgh's 'Plants of the Coast of Coromandel': dates of publication of Volume 3. Notes Roy. Bot. Gard. Edinburgh 29: 211-212. 